\title{
Oxidative stress creates a unique, CaMKII-mediated substrate for atrial fibrillation in heart failure
}

Shin Yoo, ${ }^{1}$ Gary Aistrup, ${ }^{1}$ Yohannes Shiferaw, ${ }^{2}$ Jason Ng, ${ }^{1}$ Peter J. Mohler, ${ }^{3}$ Thomas J. Hund, ${ }^{3}$ Trent Waugh, ${ }^{1}$ Suzanne Browne, ${ }^{1}$ Georg Gussak, ${ }^{1}$ Mehul Gilani, ${ }^{1}$ Bradley P. Knight, ${ }^{1}$ Rod Passman, ${ }^{1}$ Jeffrey J. Goldberger, ${ }^{1}$ J. Andrew Wasserstrom, ${ }^{1}$ and Rishi Arora'

${ }^{1}$ Feinberg Cardiovascular Research and Renal Institute, Northwestern University-Feinberg School of Medicine, Chicago, Illinois, USA. 'Department of Physics, California State University, Northridge, California, USA. ${ }^{3}$ Dorothy M. Davis Heart and Lung Research Institute, Ohio State University College of Medicine, Columbus, Ohio, USA.

The precise mechanisms by which oxidative stress (OS) causes atrial fibrillation (AF) are not known. Since AF frequently originates in the posterior left atrium (PLA), we hypothesized that $0 S$, via calmodulin-dependent protein kinase II (CaMKII) signaling, creates a fertile substrate in the PLA for triggered activity and reentry. In a canine heart failure (HF) model, OS generation and oxidizedCaMKII-induced (Ox-CaMKII-induced) RyR2 and $\mathrm{Na}_{\mathrm{v}} 1.5$ signaling were increased preferentially in the PLA (compared with left atrial appendage). Triggered $\mathrm{Ca}^{2+}$ waves (TCWs) in HF PLA myocytes were particularly sensitive to acute ROS inhibition. Computational modeling confirmed a direct relationship between $0 \mathrm{~S} / \mathrm{CaMKII}$ signaling and TCW generation. CaMKII phosphorylated $\mathrm{Na}_{\mathrm{v}} 1.5$ (CaMKII-p-Na 1.5 [S571]) was located preferentially at the intercalated disc (ID), being nearly absent at the lateral membrane. Furthermore, a decrease in ankyrin-C (AnkC) in HF led to patchy dropout of CaMKII-p-Na 1.5 at the ID, causing its distribution to become spatially heterogeneous; this corresponded to preferential slowing and inhomogeneity of conduction noted in the HF PLA. Computational modeling illustrated how conduction slowing (e.g., due to increase in CaMKII-p$\mathrm{Na}_{v}$ 1.5) interacts with fibrosis to cause reentry in the PLA. We conclude that $\mathrm{OS}$ via CaMKII leads to substrate for triggered activity and reentry in HF PLA by mechanisms independent of but complementary to fibrosis.

Conflict of interest: RA has ownership interest in Rhythm Therapeutics and receives research support from BiosenseWebster and Johnson \& Johnson.

License: Copyright 2018, American Society for Clinical Investigation.

Submitted: February 28, 2018 Accepted: September 27, 2018 Published: November 2, 2018

\section{Reference information:} JCI Insight. 2018;3(21):e120728. https://doi.org/10.1172/jici. insight.120728.

\section{Introduction}

Atrial fibrillation (AF) is a cause of significant morbidity and mortality (1). Unfortunately, traditional pharmacological therapies have had limited success in $\operatorname{AF}(2,3)$. Ablation procedures developed in recent years have been moderately successful in patients with structurally normal hearts but have suboptimal efficacy in structural heart disease (e.g., heart failure [HF]; refs. 4, 5). In order to improve upon current ablation techniques and develop new AF therapies, recent research has attempted to better define the mechanisms underlying $\mathrm{AF}$, including the mechanisms by which AF originates in the pulmonary veins (PVs) and adjoining posterior left atrium (PLA). One such mechanism is thought to be oxidative stress (OS) $(6,7)$, with ROS thought to modify the function of key ion channels and $\mathrm{Ca}^{2+}$ cycling proteins (8-10), as well as activate profibrotic signaling $(11,12)$. How OS specifically contributes to electrophysiological remodeling in the PLA - and thereby to a vulnerable AF substrate - is not known. It is known, however, that NADPH oxidase (NOX2) is a major enzymatic source of ROS in the fibrillating atrium (13). It is also known that, in patients with AF, there is an increase in atrial expression levels of methionine-oxidized CaMKII (methionine-Ox-CaMKII) (14). This form of CaMKII that is enzymatically active in the absence of $\mathrm{Ca}^{2+} / \mathrm{CaM}$, has been reported to affect the activity of both the $\mathrm{Na}^{+}$and $\mathrm{Ca}^{2+}$ currents $\left(I_{\mathrm{Na}}\right.$ and $\left.I_{\mathrm{CaI}}\right)$ involved in cardiac depolarization, as well as activity of ryanodine receptor 2 (RyR2), which is integral to excitation-contraction (E-C) coupling (15).

Since AF triggers frequently arise in the PVs/PLA, we hypothesized that OS creates a vulnerable substrate for AF in a canine HF model of AF by affecting both E-C coupling and cardiac depolarization in the PLA and, thereby, creating conditions for both triggered activity and reentry. Recent studies suggest that CaMKII-dependent RyR2 phosphorylation at serine 2814 (CaMKII-p-RyR2 [S2814]) leads to increased 
propensity for spontaneous calcium $\left(\mathrm{Ca}^{2+}\right)$ release (in the form of $\mathrm{Ca}^{2+}$ waves) in atrial myocytes of patients with chronic AF (16). Our work indicates that atrial myocytes in the setting of HF have increased susceptibility to the development of $\mathrm{Ca}^{2+}$ waves during rapid atrial pacing (i.e., triggered $\mathrm{Ca}^{2+}$ waves; TCWs) (17). $\mathrm{Ca}^{2+}$ waves are known to create conditions in both the ventricle and atrium for the genesis of afterdepolarizations, which can lead to triggered activity and/or dispersion of repolarization $(18,19)$. Another major mechanism thought to underlie the development of a vulnerable AF substrate in HF is slow and inhomogeneous conduction in the intact atrium, which creates conditions for reentry (20-22). While several studies suggest that increased fibrosis in the HF atrium is a major cause of this inhomogeneous conduction (21, 23), it is also known that the HF atrium undergoes significant electrical remodeling (24), with alterations in the $I_{\mathrm{Na}}$ having been implicated in some studies in creation of the AF disease state (25). Our specific hypotheses for this study were, therefore, as follows: (a) OS generation - and downstream oxidation of key signaling proteins - is preferentially increased in the PLA/PVs in the HF atrium; (b) the increased vulnerability of $\mathrm{HF}$ atrial myocytes to TCW generation is at least partially mediated by OS, with OS creating substrate for TCWs by increasing CaMKII-p-RyR2 (S2814) in the HF PLA; and (c) OS, via Ox-CaMKII, increases the level of CaMKII phosphorylated $\mathrm{Na}_{\mathrm{v}} 1.5$ in HF PLA. This increase in CaMKII phosphorylated $\mathrm{Na}_{\mathrm{v}} 1.5$, particularly CaMKII-p-Na 1.5 (S571) $(15,26)$, creates conditions for slow and inhomogeneous conduction in the HF PLA, which - in the presence of existing fibrosis - enhances substrate for reentry (in the PLA).

Our results demonstrate that OS - acting at least in part through increased constitutive activity of CaMKII - creates a highly vulnerable substrate within the HF PLA for both triggered activity and reentry that promotes AF beyond fibrosis. These data not only shed light on the mechanisms underlying the creation of a vulnerable AF substrate, but they also have potentially important translational implications, especially with regard to the design of new, mechanism-guided therapies for AF. These therapies may include novel, gene-based approaches targeted at enzymatic sources of OS (e.g., NOX2), as well as downstream signaling mediators of OS-induced electrophysiological remodeling in the atrium (e.g., CaMKII).

\section{Results}

\section{Preferential increase in $\mathrm{O}_{2}^{-}$generation and protein oxidation in HF PLA}

Regional differences in $\mathrm{O}_{2}^{-}$generation and carbonylation within left atrium. We first investigated whether or not there was greater $\mathrm{O}_{2}^{-}$generation and possible regional differences within the left atrium in the setting of HF. Lucigenin chemiluminescence assay revealed a significant increase in overall $\mathrm{O}_{2}^{-}$generation in $\mathrm{HF}$ left atrial homogenate, compared with control samples (Figure 1A). This increase in $\mathrm{O}_{2}^{-}$generation occurred preferentially in the PLA, with there being no significant increase in $\mathrm{O}_{2}^{-}$generation in the left atrial appendage (LAA) (Figure 1B). Next, we determined relative contribution to $\mathrm{O}_{2}^{-}$generation by various enzymatic sources of ROS (by the application of specific ROS inhibitors). There was higher activity mitochondrial ROS in HF PLA, compared with control PLA, with NOX2-generated ROS showing a trend toward an increase $(P=0.140)$ (Figure 1C). No significant difference between control LAA and HF LAA was noted for any enzymatic sources of ROS (Figure 1D). Consistent with higher $\mathrm{O}_{2}^{-}$generation in HF PLA, immunoblot analysis showed that expression of gp91, a major subunit of NOX2, was also greater in $\mathrm{HF}$ atria, compared with control (Figure 1E). In view of increased generation of $\mathrm{O}_{2}^{-}$in the HF PLA, we next sought to determine if higher ROS generation in HF PLA could affect downstream molecules by either direct protein oxidation or indirectly via increased activity of protein kinase signaling pathways. To address the possibility of direct modification, we assessed carbonylation in control and $\mathrm{HF}$ atrial samples. Level of carbonylation was higher in $\mathrm{HF}$, compared with control (Figure 1F). Furthermore, there was a preferential increase in carbonylation in the HF PLA compared with HF LAA (Figure $1 \mathrm{G})$, which is consistent with higher levels of ROS generation found earlier in the HF PLA.

Differences in oxidation between left and right atrium. To determine whether there were differences in oxidation between the left and right atrium, we examined relative differences in carbonylation between the LAA and the right atrial appendage (RAA) in dogs with HF. As shown in Figure 1H, in HF atria, the LAA is significantly more oxidized than the RAA. Taken together, these data further support our overall conclusion that OS appears to be preferentially increased in the HF PLA as compared with other regions of the atria.

Regional differences in expression and spatial distribution of Ox-CaMKII within the left atrium. Since direct modification of cellular proteins by ROS can be spatiotemporally limited, we also assessed ROS-induced oxidation of CaMKII. Immunoblot analysis revealed a significant increase in the ratio of oxidized-CaMKII (Ox-CaMKII) to total CaMKII in HF PLA, compared with control PLA, but not in HF LAA vs. control 
A

Overall $\mathrm{O}_{2}^{-}$generation

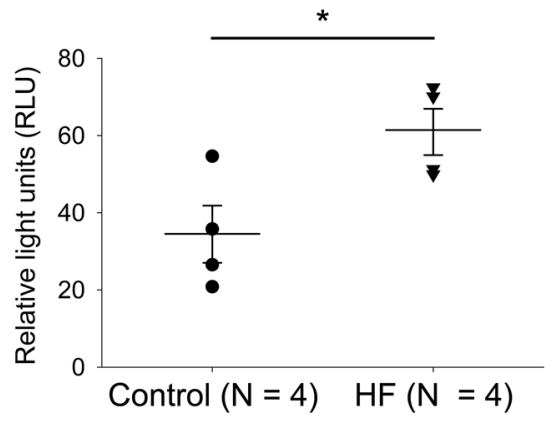

C $\mathrm{O}_{2}^{-}$generation in PLA

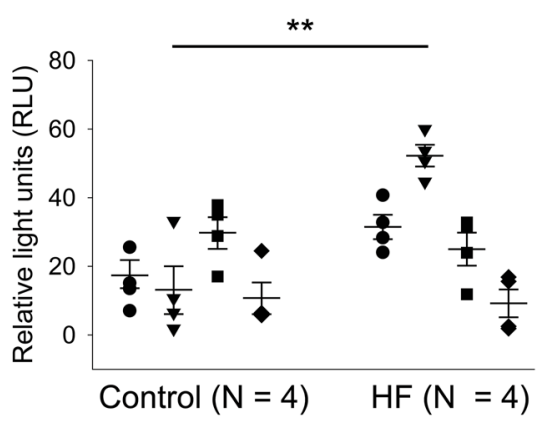

E $91 \mathrm{kd}$

$38 \mathrm{kd}$

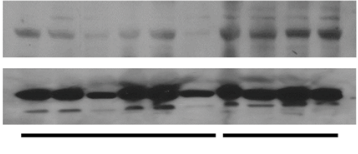

Control

$\mathrm{HF}$

Relative gp91 expression

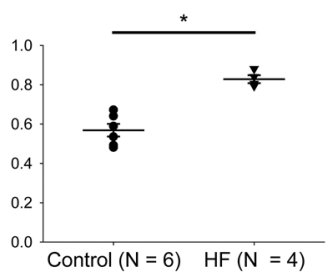

gp91

GAPDH

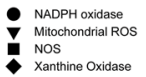

Mitochondrial ROS
NOS

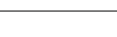

\section{.}


HF RAA. Data are represented as mean \pm SEM; ${ }^{*} P<0.05,{ }^{* *} P<0.01$. (A, E, F, G, and $\left.\mathbf{H}\right)$ Independent $t$ test. (B) Two-way ANOVA test of main effects. (C and D) Two-way ANOVA with Bonferroni correction on comparison of HF vs. control within each enzyme. HF, heart failure; PLA, posterior left atrium; LAA, left atrial appendage; RAA, right atrial appendage. See complete unedited blots in the supplemental material.

LAA (Figure 2A). Ox-CaMKII has been suggested to effect conduction slowing in the ventricle after myocardial infarction (27). Since slow and inhomogeneous conduction are thought to promote an AF substrate in $\operatorname{HF}(23,28)$, we hypothesized that the spatial distribution of Ox-CaMKII would be more heterogeneous in HF compared with control PLA. We performed a large-scale IHC analysis in entire tissue sections to determine spatial distribution of Ox-CaMKII. Consistent with the immunoblot data, quantification of immunofluorescence intensity of Ox-CaMKII (green) revealed not only higher intensity of Ox-CaMKII (control PLA, $63.45 \pm 4.47 \mathrm{AU}$; HF PLA, $91.51 \pm 3.61 \mathrm{AU} ; P<0.001$ ), but also more spatially heterogeneous distribution of Ox-CaMKII, compared with control PLA (Figure 2B). There was no significant difference in spatial distribution of Ox-CaMKII in HF LAA vs. control LAA (Supplemental Figure 1A; supplemental material available online with this article; https://doi.org/10.1172/jci.insight.120728DS1).

\section{Increased incidence of TCWs in HF PLA myocytes and attenuation of TCWs by} mitochondrial ROS inhibition

Aberrant $\mathrm{Ca}^{2+}$ cycling is thought to contribute to AF substrate formation (29). We recently described a unique form of aberrant sarcoplasmic reticulum (SR) $\mathrm{Ca}^{2+}$ release in atrial myocytes, wherein atrial myocytes demonstrate the presence of $\mathrm{Ca}^{2+}$ waves that occur during rapid atrial stimulation, not during diastole (17). Preliminary data indicate that these TCWs cause incomplete cellular repolarization on a beat-to-beat basis and, therefore, could contribute to afterdepolarizations and/or dispersion of repolarization (30). The incidence of TCWs increases significantly in $\mathrm{HF}$ myocytes, with $\mathrm{Ca}^{2+}$ waves occurring at significantly longer cycle lengths than in normal myocytes (17). Since CaMKII-p-RyR2 (S2814) has been shown to alter SR $\mathrm{Ca}^{2+}$ release (16), and as Ox-CaMKII - which is known to be enzymatically active in the absence of $\mathrm{Ca}^{2+}$ / CaM - was increased in the HF PLA, we measured the level of CaMKII-p-RyR2 (S2814). The ratio of CaMKII-p-RyR2 (S2814) to total RyR2 was significantly higher in HF PLA, compared with control PLA (Figure 3A; see complete unedited blots in the supplemental material; 2 exposures of the complete gel have been provided, as the fourth sample in the HF PLA is best seen on the longer exposure). There was no difference in ratio of CaMKII-p-RyR2 (S2814) to total RyR2 in the HF LAA vs. control LAA.

Since both enhanced OS and enhanced CaMKII phosphorylation of RyR2 have been shown to alter $\mathrm{SR} \mathrm{Ca}^{2+}$ release in ventricular and atrial myocytes $(16,31)$, we hypothesized that the increased levels Ox-CaMKII and the ratio of CaMKII-p-RyR2 (S2814) to total RyR2 noted in the HF PLA would be associated with: (a) an increased propensity for HF PLA myocytes to develop TCWs, as compared with LAA myocytes, and (b) acute inhibition of ROS to inhibit TCW generation in HF atrial myocytes.

Quantification of TCWs evoked by field stimulation in freshly isolated HF atrial myocytes did indicate a higher incidence of TCWs compared with normal atrial myocytes at a basic cycle length (BCL) of $200 \mathrm{~ms}$ (Figure 3B). In addition, the incidence of TCWs was significantly greater in HF PLA compared with HF LAA myocytes at $300 \mathrm{~ms}$ BCL (Figure 3C).

To address whether or not the higher incidence of TCWs in HF PLA is ROS sensitive, we measured $\mathrm{Ca}^{2+}$ cycling following application of inhibitors for the different enzymatic sources of ROS in HF PLA and HF LAA myocytes. Figure 3D shows that the frequency of TCWs in PLA myocytes was significantly attenuated by preincubation with mitochondrial ROS scavenger, mito-TEMPO, as well as by a NOX2 inhibitor, apocynin. Even though apocynin is not a specific inhibitor of NOX2, our data - when taken together with the observed increase of NOX2-generated $\mathrm{O}_{2}^{-}$and gp91 in the HF PLA - does indicate a likely role for NOX2 in TCW generation in HF atrial myocytes. We also investigated the characteristics of $\mathrm{Ca}^{2+}$ transients. Given that TCWs typically manifest before $\mathrm{Ca}^{2+}$ transients decay to baseline (before full repolarization/relaxation), analyses of $\mathrm{Ca}^{2+}$ transient characteristics were restricted to pacing cycle lengths not containing TCWs. Such assessment of multiple spatiotemporal properties of $\mathrm{Ca}^{2+}$ transients indicated statistically insignificant differences between untreated vs. mito-TEMPO- or apocynin-treated myocytes (Supplemental Figures 2 and 3). Since the level of CaMKII-p-RyR2 (S2814) was preferentially elevated in the PLA, we also assessed whether TCWs in HF PLA myocytes were sensitive to CaMKII inhibition. Supplemental Figure 4 shows that preincubation with KN-93, a CaMKII inhibitor, attenuated the incidence of TCWs in HF PLA myocytes. We also performed $\mathrm{Ca}^{2+}$ transient 
A

Expression of Ox-CaMKII in PLA

$55 \mathrm{kd}$
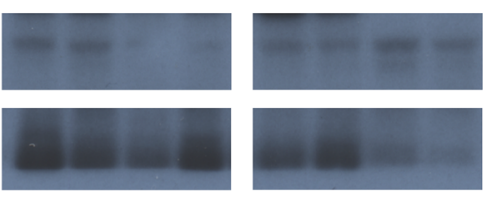

HF

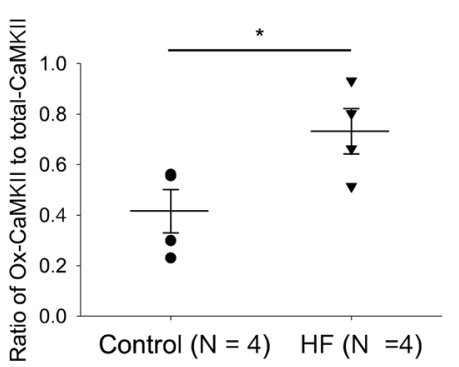

B

Control

PLA
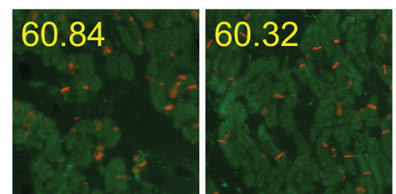

61.79
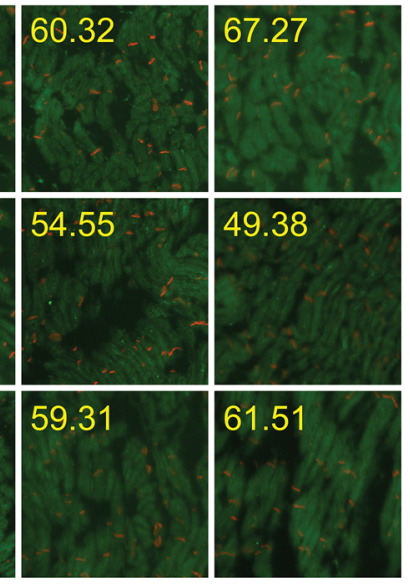

Expression of Ox-CaMKII in LAA

Ox-CaMKII

Total-CaMKII

(1)

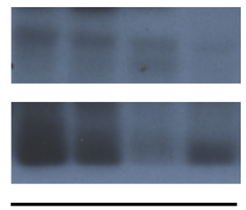

Control

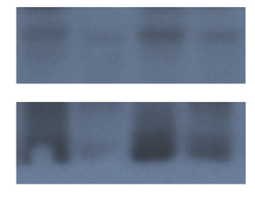

$55 \mathrm{kd}$

HF

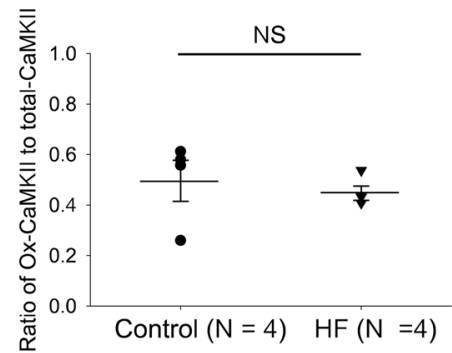

HF

PLA
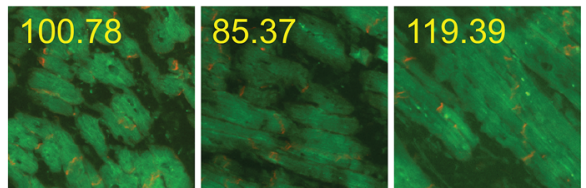

Heterogeneity of spatial distribution of Ox-CaMKI

Coefficient of variation of Ox-CaMKII immunofluorescence in PLA
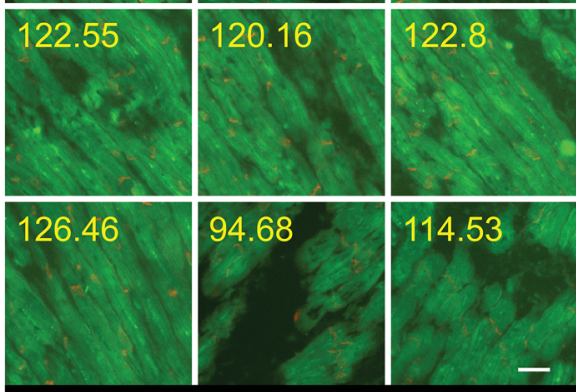

94.68

114.53

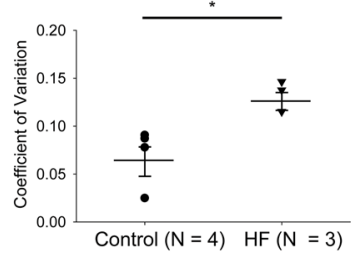

Ox-CaMKII Cadherin

Figure 2. Increase in expression and increased heterogeneity of spatial distribution of Ox-CaMKII in HF PLA. (A) Representative immunoblot and densitometric measurements of Ox-CaMKII (normalized to native CaMKII) from control and HF in PLA (left) and LAA (right). (B) Quantification of immunofluorescence in all myocytes in random nine 10x images in control and HF PLA. Coefficient of variation of Ox-CaMKII immunofluorescence for control vs. HF PLA is compared in the graph on the right. Scale bar: $40 \mu \mathrm{m}$. Data are represented as mean $\pm \mathrm{SEM}$. ${ }^{*} P<0.05$. Independent $t$ tests. HF, heart failure; PLA, posterior left atrium; LAA, left atrial appendage. See complete unedited blots in the supplemental material.

analysis in the presence of KN93. The only significant change induced by KN93 was the width of the transient at $50 \%$ of its magnitude (TW50, Supplemental Figure 5).

ROS leads to generation of TCWs via CaMKII signaling - a computational modeling study To better understand the connection between OS, CaMKII signaling, and TCW generation in HF PLA myocytes, we investigated the effect of OS on $\mathrm{Ca}^{2+}$ handling using a spatially distributed atrial cell model. To model the relationship between ROS and CaMKII, we applied an established mathematical model by Christensen et al. (32), which includes an oxidized active state of CaMKII that is regulated by ROS activity. The subsequent effect of CaMKII on $\mathrm{Ca}^{2+}$ cycling activity was then described using a model of RyR phosphorylation by Hashambhoy et al. (33). Using this combined approach, we then explored the 
A

Expression of $p-R y R 2$ in PLA

$560 \mathrm{k}$

$560 \mathrm{k}$
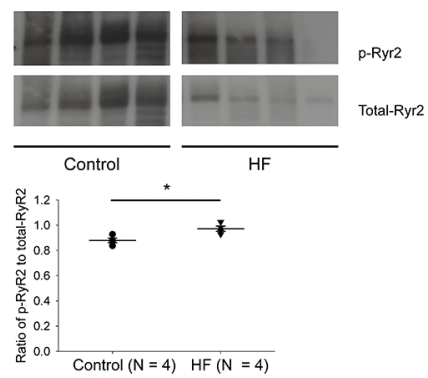

Expression of $p-R y R 2$ in LAA

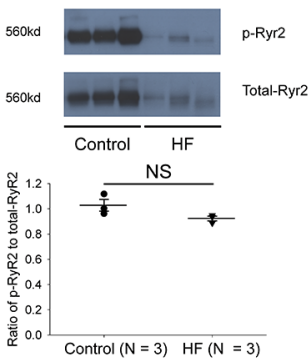

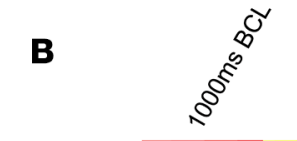
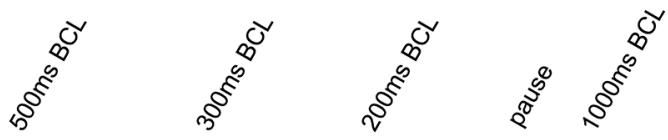

Basic cycle length

$1000 \mathrm{~ms}$

$500 \mathrm{~ms}$

$300 \mathrm{~ms}$

$200 \mathrm{~ms}$

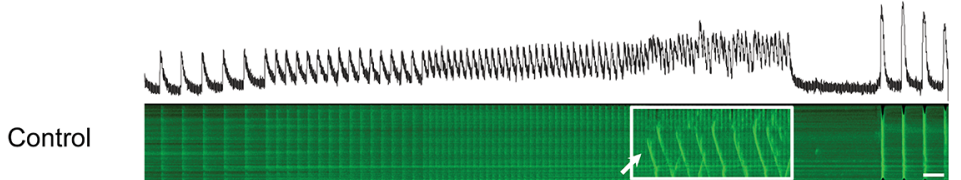

Triggered $\mathrm{Ca}^{2+}$ wave

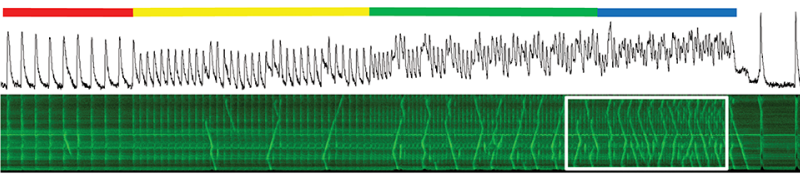

$\mathrm{Ca}^{2+}$ wave frequency (BCL @ 200 ms)

Control

HF
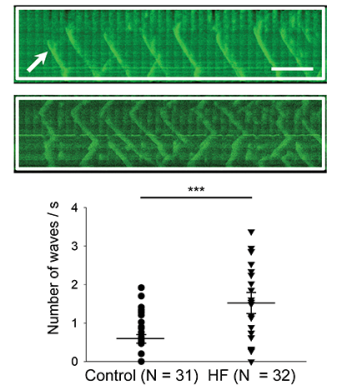

\section{C}

HF PLA

HF LAA

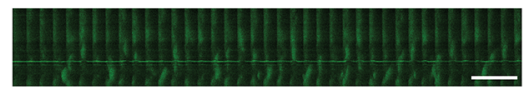

$\mathrm{Ca}^{2+}$ wave frequency in HF PLA vs. HF LAA

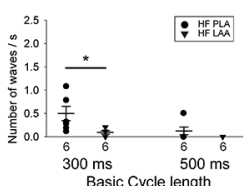

$300 \mathrm{~ms}$ BCL

D

Control
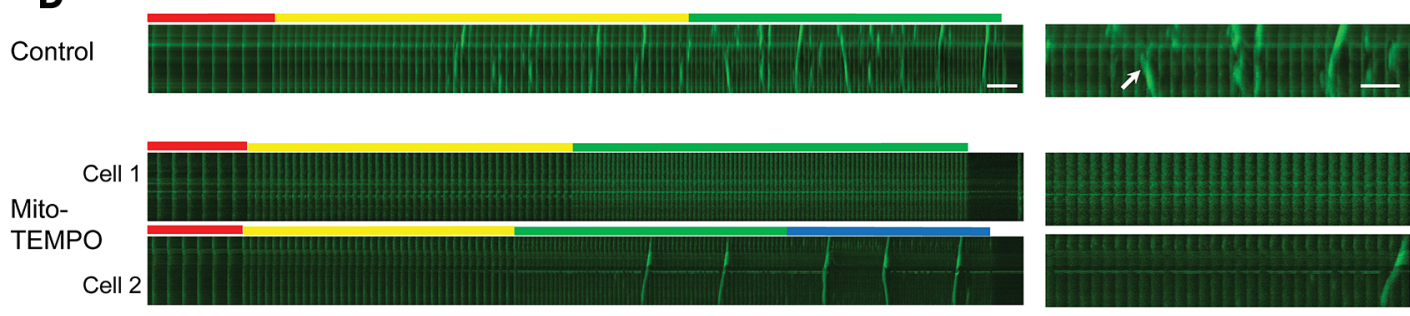

Apocynin
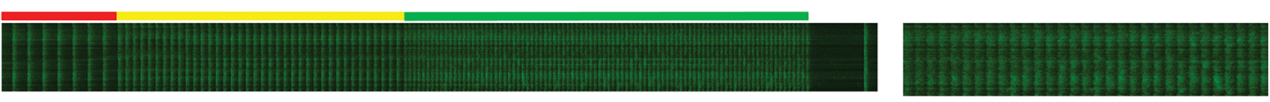

$300 \mathrm{~ms} \mathrm{BCL}$

$\mathrm{Ca}^{2+}$ wave frequency in PLA

$\mathrm{Ca}^{2+}$ wave frequency in LAA
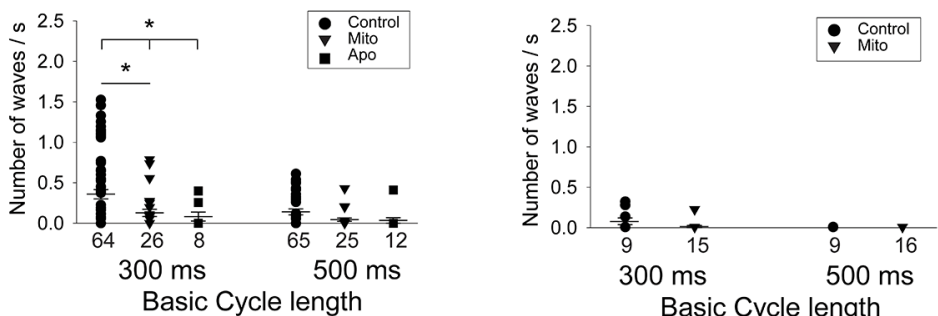
Figure 3. Increased expression of CaMKII-p-RyR2 (S2814) in HF PLA and sensitivity of triggered Ca ${ }^{2+}$ waves in PLA myocytes to acute ROS inhibition. (A) Representative immunoblot and densitometric measurements of CaMKII-p-RyR2 (S2814) (normalized to native RyR2) from control and HF in PLA (left) and LAA (right). (B) HF atrial myocytes showed higher incidence of triggered $\mathrm{Ca}^{2+}$ waves, compared with control. (C) Higher incidence of triggered Ca ${ }^{2+}$ waves in HF PLA myocytes, compared with HF LAA myocytes at 300 ms BCL. (D) $\mathrm{Ca}^{2+}$ imaging showed attenuation of incidence of triggered $\mathrm{Ca}^{2+}$ waves at $500 \mathrm{~ms}$ and $300 \mathrm{~ms} \mathrm{BCL}$ in the presence of mito-TEMPO and apocynin in HF PLA myocytes, but not in HF LAA myocytes. Time bar: 1 second. The myocytes for these experiments were obtained from $4 \mathrm{HF}$ dogs, and the number of myocytes for each experimental condition is given in each figure panel. Data are represented as mean \pm SEM. ${ }^{*} P<0.05$, ${ }^{* *} P<0.001$. (A and B) Independent $t$ test. (C) Paired $t$ test. (D) Main effect of ANOVA ( $P=0.017$ at 300 ms, 0.053 at $500 \mathrm{~ms}$ ), post hoc $t$ tests with each cycle with Bonferroni correction. HF, heart failure; PLA, posterior left atrium; LAA, left atrial appendage. See complete unedited blots in the supplemental material.

relationship between ROS activity and the spatiotemporal dynamics of $\mathrm{Ca}^{2+}$ cycling in atrial cells. As a starting point, we first considered the steady state $\mathrm{Ca}^{2+}$ transient for a range of ROS concentrations. In Figure $4 \mathrm{~A}$, we show the $\mathrm{Ca}^{2+}$ transient after 30 beats of pacing with periodic stimulation in which the ROS concentration was fixed at low concentrations of ROS (i.e., ROS $=1 \mu \mathrm{M}$ ). Here, we see that the $\mathrm{Ca}^{2+}$ transient was synchronized with the periodic pacing. To keep track of subcellular $\mathrm{Ca}^{2+}$, we also simulated a longitudinal line scan that measured the dyadic junction $\mathrm{Ca}^{2+}$ concentration within our $60 \times 20 \times 20$ atrial myocytes model (Supplemental Figure 6). The result is that subcellular $\mathrm{Ca}^{2+}$ rises mostly on the cell boundary, and there was minimal $\mathrm{Ca}^{2+}$ release in the cell interior. In contrast, we also performed a similar simulation where CaMKII was oxidized with ROS $=3 \mu \mathrm{M}$ (Figure 4B) and with ROS $=5 \mu \mathrm{M}$ (Figure $4 \mathrm{C})$. In this case, we found that, after several beats, $\mathrm{Ca}^{2+}$ release occurred in the cell interior in the form of mini-waves. At intermediate levels of ROS $(3 \mu \mathrm{M})$, these waves were found to grow in magnitude, eventually turning into full $\mathrm{Ca}^{2+}$ waves spanning the entire longitudinal axis of the atrial myocytes at higher concentrations of ROS $(5 \mu \mathrm{M})$. This effect appears to be due to the increased "leakiness" of the RyR2 because of constitutive activity of CaMKII resulting from its oxidation that led to an increased propensity of $\mathrm{Ca}^{2+}$ wave propagation in the cell interior.

Taken together, the experimental and modeling data presented above demonstrate that OS is intricately involved in the generation of TCWs in HF atrial myocytes, with CaMKII signaling being one of the likely mediators of this effect of OS generation on TCW generation.

CaMKII-dependent phosphorylation of $\mathrm{Na}_{\mathrm{v}} 1.5$ is preferentially increased in HF PLA and localized at the intercalated disc (ID) in atrial myocytes

CaMKII phosphorylates $\mathrm{Na}_{\mathrm{v}} 1.5$, resulting in altered $I_{\mathrm{Na}}$ steady-state inactivation, recovery from inactivation, and late current. CaMKII-p-Na 1.5 (S571) enhances late $\mathrm{Na}^{+}$current and decreases channel availability $(15,26)$. Recent studies suggest that $\mathrm{Na}^{+}$channels exist in more than one spatial pool in the cell membrane, owing to the interactions of $\mathrm{Na}_{\mathrm{v}} 1.5$ with a variety of kinases (including CaMKII) and binding protein partners that help the trafficking of the $\mathrm{Na}^{+}$channel macromolecular complex to the cell membrane $(26,34)$. As mentioned earlier, HF leads to a vulnerable substrate for AF, at least in part by leading to slowing and inhomogeneity of conduction in the atrium. Since CaMKII, via its interactions with binding partners such as AnkG and $\beta I V$-spectrin, is thought to associate with $\mathrm{Na}_{\mathrm{v}} 1.5$ at the ID, we hypothesized that CaMKII-p-Na 1.5 (S571) has a unique pattern of expression and spatial distribution in the HF atrium that likely contributes to the slow and inhomogeneous conduction observed in HF.

Consistent with the increased expression of Ox-CaMKII in HF PLA, we found that the ratio of CaMKII-p-Na 1.5 (S571) to total $\mathrm{Na}_{\mathrm{v}} 1.5$ was higher in HF PLA, compared with control PLA (Figure 5A). Of note, there was a significant decrease in native $\mathrm{Na}_{\mathrm{v}} 1.5$ expression in the $\mathrm{HF}$ atrium (Figure $5 \mathrm{~A}$ ). There was no significant difference in the ratio of CaMKII-p-Na 1.5 (S571) to total $\mathrm{Na}_{\mathrm{v}} 1.5$ between control LAA and HF LAA (Supplemental Figure 7A).

To assess subcellular localization of CaMKII-p- $\mathrm{Na}_{\mathrm{v}} 1.5$ (S571) in control and HF atrium, we performed IHC with confocal microscopy. As expected, native $\mathrm{Na}_{\mathrm{v}} 1.5$ was located at both the ID and lateral membrane in control atrial myocytes, as well as in $\mathrm{HF}$ atrial myocytes (Figure 5B, top panel, and Supplemental Figure 7B). In sharp contrast, CaMKII-p-Na 1.5 (S571) was localized preferentially at the ID and was almost entirely absent from the lateral membrane in control atrial myocytes (only a negligible number of myocytes showed CaMKII-p-Na 1.5 [S571] labeling at the lateral membrane; Figure 5B, middle panel, and Figure $5 \mathrm{C}$ ). This absence of CaMKII-p-Na 1.5 (S571) at the lateral membrane was observed in both control and $\mathrm{HF}$ atrium (Figure 5B, bottom panel). To our knowledge, this is the first report of preferential localization of CaMKII-p-Na 1.5 (S571) at only 1 spatial pool. The near-complete absence of CaMKII-p-Na 1.5 (S571) may have major implications for slow and/or inhomogeneous conduction in the HF atrium, in view of data 
A

$\mathrm{ROS}=1 \mu \mathrm{M}$
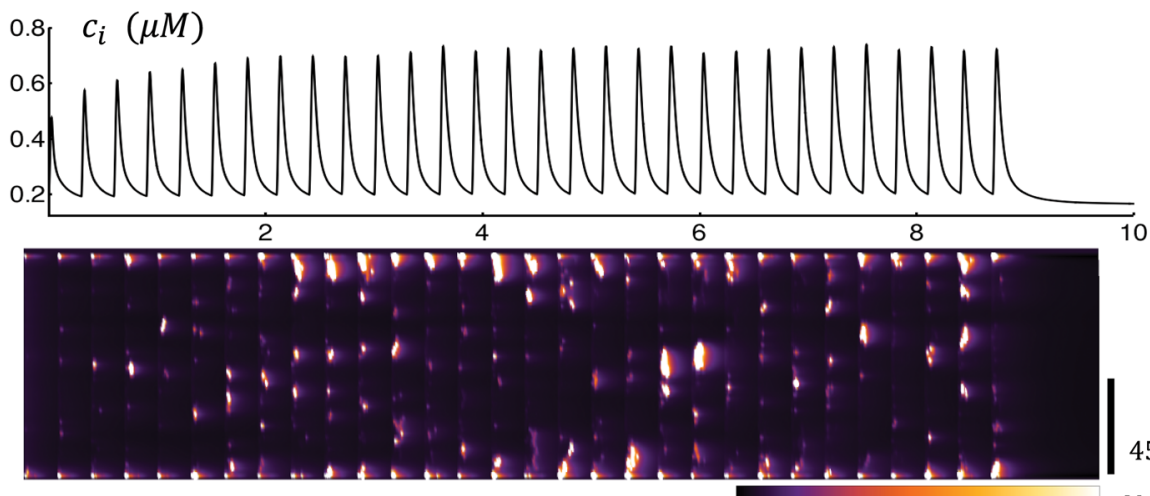

I

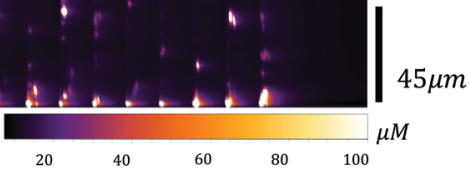

B

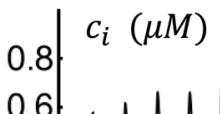

0.6

0.4 0.2

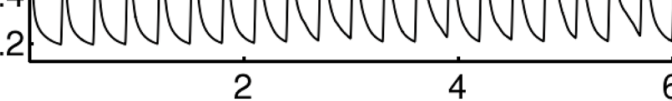

$\mathrm{ROS}=3 \mu \mathrm{M}$

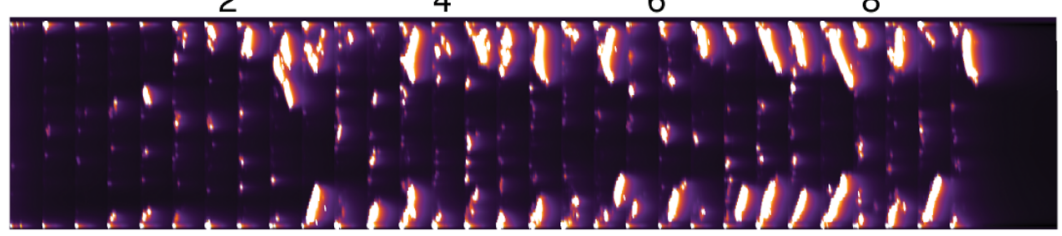

$t(s)$
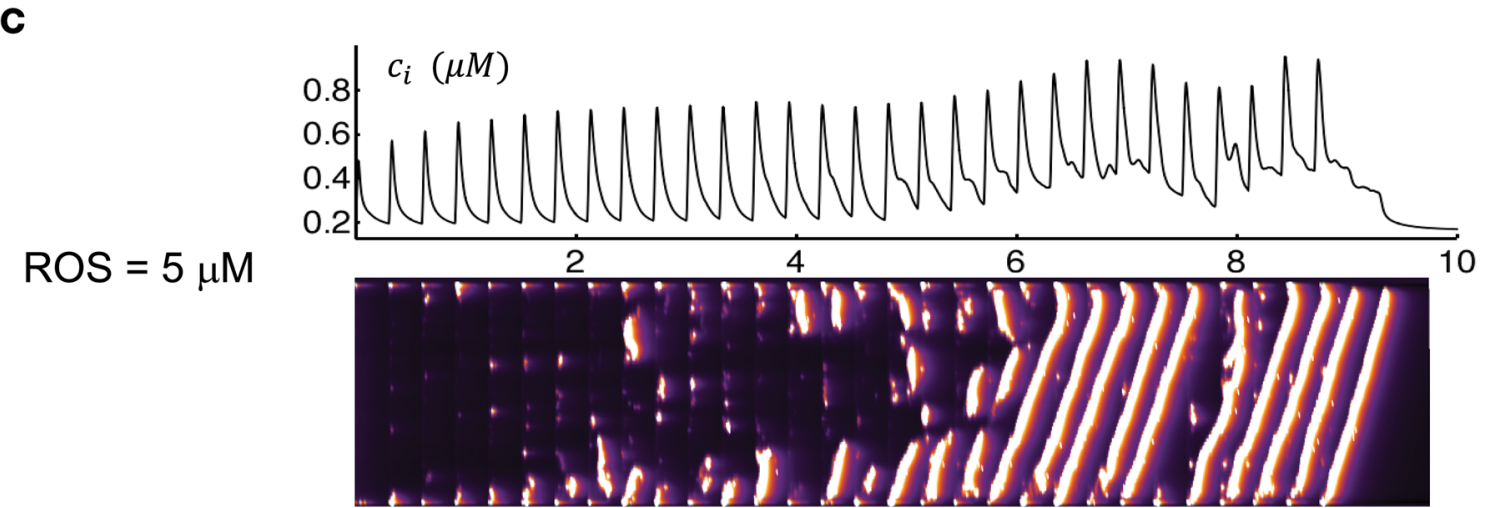

$t(s)$

Figure 4. Computational modeling of $\mathrm{Ca}^{2+}$ cycling at low and high level of ROS. Computational modeling of $\mathrm{Ca}^{2+} \mathrm{cycling}$ at low level of ROS (A), intermediate level of ROS (B), and high level of ROS (C). Top trace represents the $\mathrm{Ca}^{2+}$ transient when the cell is paced for 30 beats using a periodic voltage clamp. Bottom trace is a longitudinal linescan of our spatially distributed model of $60 \times 20 \times 20$ calcium release units. The linescan location is at position $n_{\mathrm{y}}=10$ and $n_{\mathrm{z}}=15$. Scale bar: $45 \mu \mathrm{m}$.

in a $\mathrm{KO}$ mouse model wherein the absence of $\mathrm{Na}_{v} 1.5$ at the lateral membrane leads to at least a $40 \%$ reduction in conduction velocity $(\mathrm{CV})$ in the myocardium (35). Indeed, as discussed later in this manuscript, the preferential increase in CaMKII-p-Na 1.5 (S571) noted in the HF PLA closely corresponds to the preferential slowing of conduction noted in this region in the setting of HF (as compared with the LAA).

Decrease in AnkG attenuates trafficking of CaMKII-p-Na 1.5 (S571) to the ID in HF, leading to increased spatial heterogeneity of distribution of CaMKII-p-Nav1.5 (S571) in HF

As in normal dogs, CaMKII-p-Na 1.5 (S571) was localized almost entirely at the ID in the HF atrium 
A

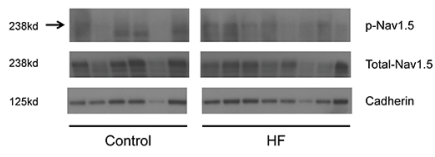

Expression of p-Nav1.5 in PLA

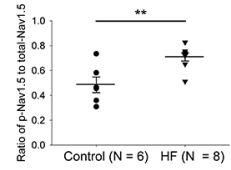

Expression of total-Nav1.5 in PLA
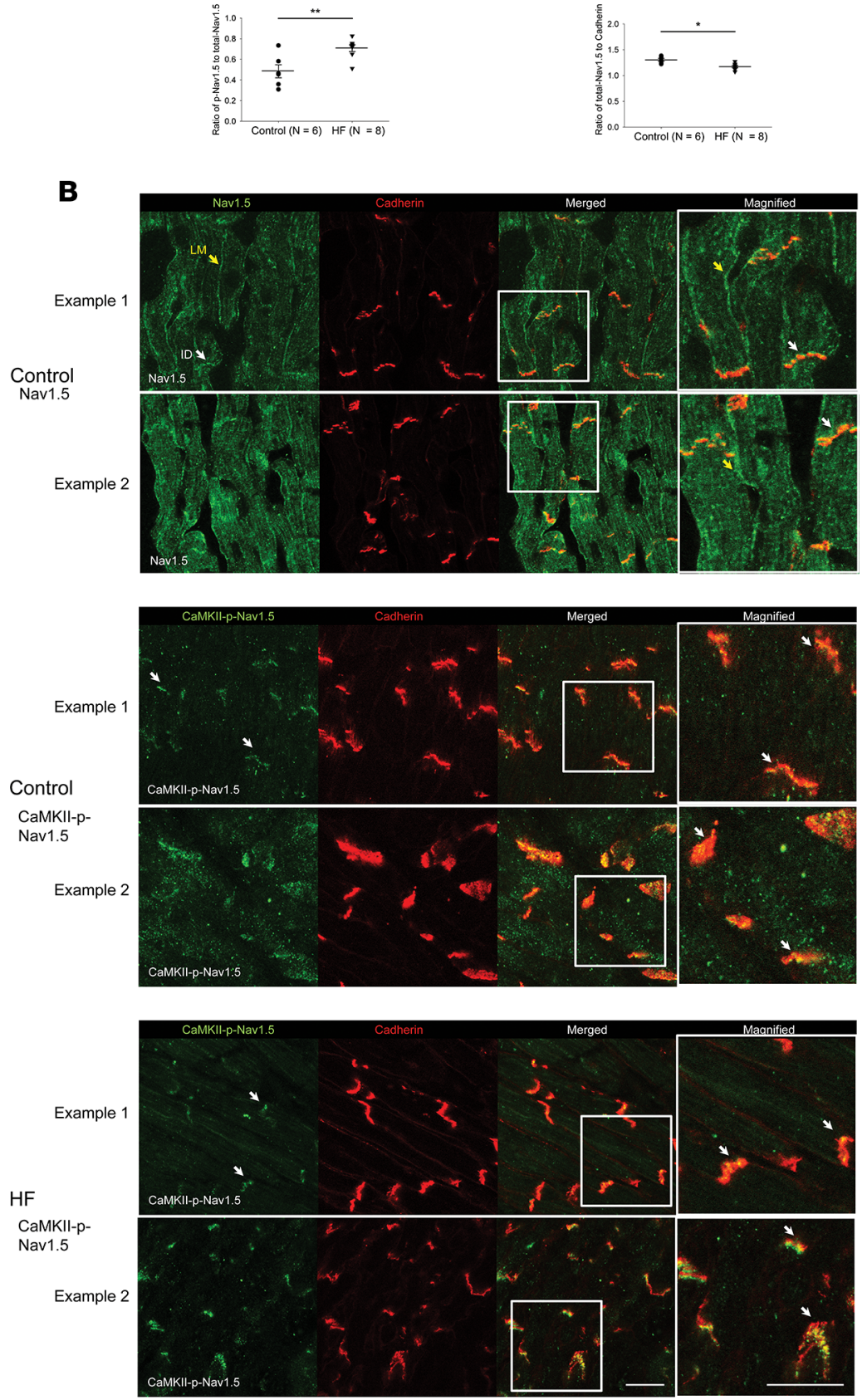

C

Myocytes with Nav1.5/p-Nav1.5 at $\mathrm{LM}$ in control and $\mathrm{HF}$

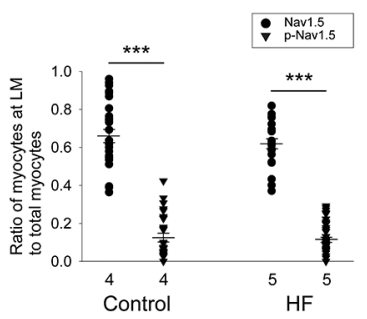

Figure 5. Increased expression of CaMKII-phosphorylated $\mathrm{Na}^{1.5}$ at serine 571 in HF PLA and preferential localization of CaMKII-p-Na 1.5 (S571) at the ID. (A) Representative immunoblot of CaMKII-p- $\mathrm{Na}_{\mathrm{v}} 1.5$ (S571), native $\mathrm{Na}_{\mathrm{v}} 1.5$, and cadherin (top) and densitometric measurements for CaMKII$\mathrm{p}-\mathrm{Na}_{v} 1.5$ (S571) (normalized to native $\mathrm{Na}_{v} 1.5$, bottom left) and native $\mathrm{Na}_{\mathrm{v}} 1.5$ (normalized to cadherin, bottom right) from control and HF in PLA. (B) While $\mathrm{Na}_{v} 1.5$ was localized at both ID (white arrows) and the lateral membrane (LM, yellow arrows) in both control and HF (top), CaMKII-p- $\mathrm{Na}_{\mathrm{v}} 1.5$ (S571) was only localized at ID in control (middle) and HF (bottom). Scale bar: $40 \mu \mathrm{m}$. (C) Quantification of myocytes with LM labeling of $\mathrm{Na}_{v} 1.5$ and CaMKII-p-Na 1.5 (S571) in control and HF myocytes. Data are represented as mean \pm SEM. ${ }^{*} P<0.05,{ }^{* *} P<0.01,{ }^{* * *} P<0.001$. (A) Independent $t$ tests. (C) Two-way ANOVA, Bonferroni adjusted within level comparisons. HF, heart failure; PLA, posterior left atrium; ID, intercalated disc. See complete unedited blots in the supplemental material.

(Figure 5B, bottom panel). Interestingly, the percentage of IDs that had demonstrated the presence of CaMKII-p-Na 1.5 (S571) was significantly decreased in HF compared with normal atrium, due to a patchy dropout of CaMKII-p-Na 1.5 (S571) at the ID (Figure 6A). The absence of CaMKII-p-Na 1.5 (S571) labeling at a significant percentage of IDs in HF, even though these IDs showed adequate cadherin labeling, translated into a significant decrease in the ratio between CaMKII-p$\mathrm{Na}_{\mathrm{v}} 1.5$ (S571) and cadherin labeling at the ID (Figure $6 \mathrm{~A})$. Some dropout of native $\mathrm{Na}_{\mathrm{v}} 1.5$ at the ID was also noted in the HF atrium (Supplemental Figure 7B).

To investigate possible mechanisms for this patchy dropout of CaMKII-p- $\mathrm{Na}_{\mathrm{v}} 1.5$ (S571) in HF, we next determined the expression levels of important binding partners to traffic $\mathrm{Na}_{\mathrm{v}} 1.5$ to the ID (i.e., AnkG and $\beta I V$-spectrin; ref. 36). Immunoblot analysis revealed a significant reduction of AnkG in $\mathrm{HF}$ atrium in both cytosolic and membrane fractions (Figure 6B) compared with control atria. Immunocytochemistry experiments further confirmed this decrease in AnkG in HF atrium, with a significant decrease in AnkG at the ID in $\mathrm{HF}$ atrial myocytes (even though cadherin labeling was similar in control and HF myocytes) (Figure 6C). Changes in expression of $\beta \mathrm{IV}$-spectrin were less pronounced, with $\beta I V$-spectrin being nearly absent in the cytosolic fraction but increased in the membrane fraction (Supplemental Figure 7C).

As shown in Figure 6D, AnkG and CaMKII-p$\mathrm{Na}_{\mathrm{v}} 1.5$ closely colocalize with each other. AnkG and CaMKII-p-Na 1.5 (S571) are either both present or both absent at the ID in the vast majority (almost 90\%) of HF atrial myocytes. Only a small minority of myocytes demonstrate the presence of only a single protein at the ID. Taken together, these results strongly suggest that a decrease in AnkG contributes to the dropout of CaMKII-p-Na 1.5 (S571) at the ID in the HF. 
A

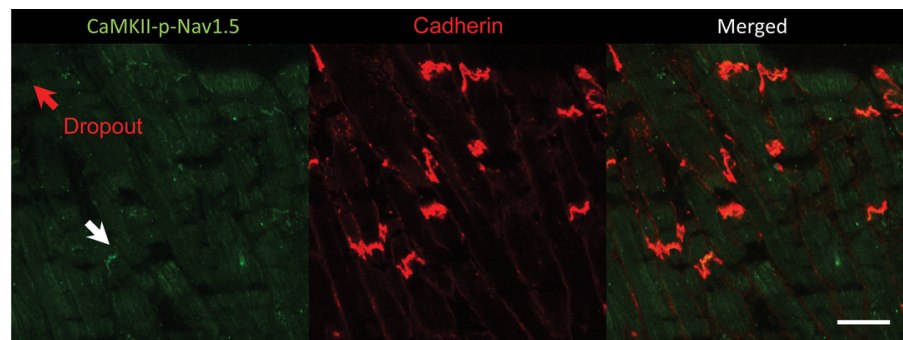

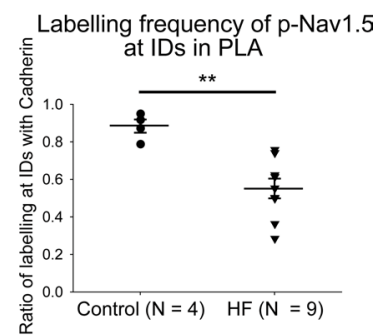

B
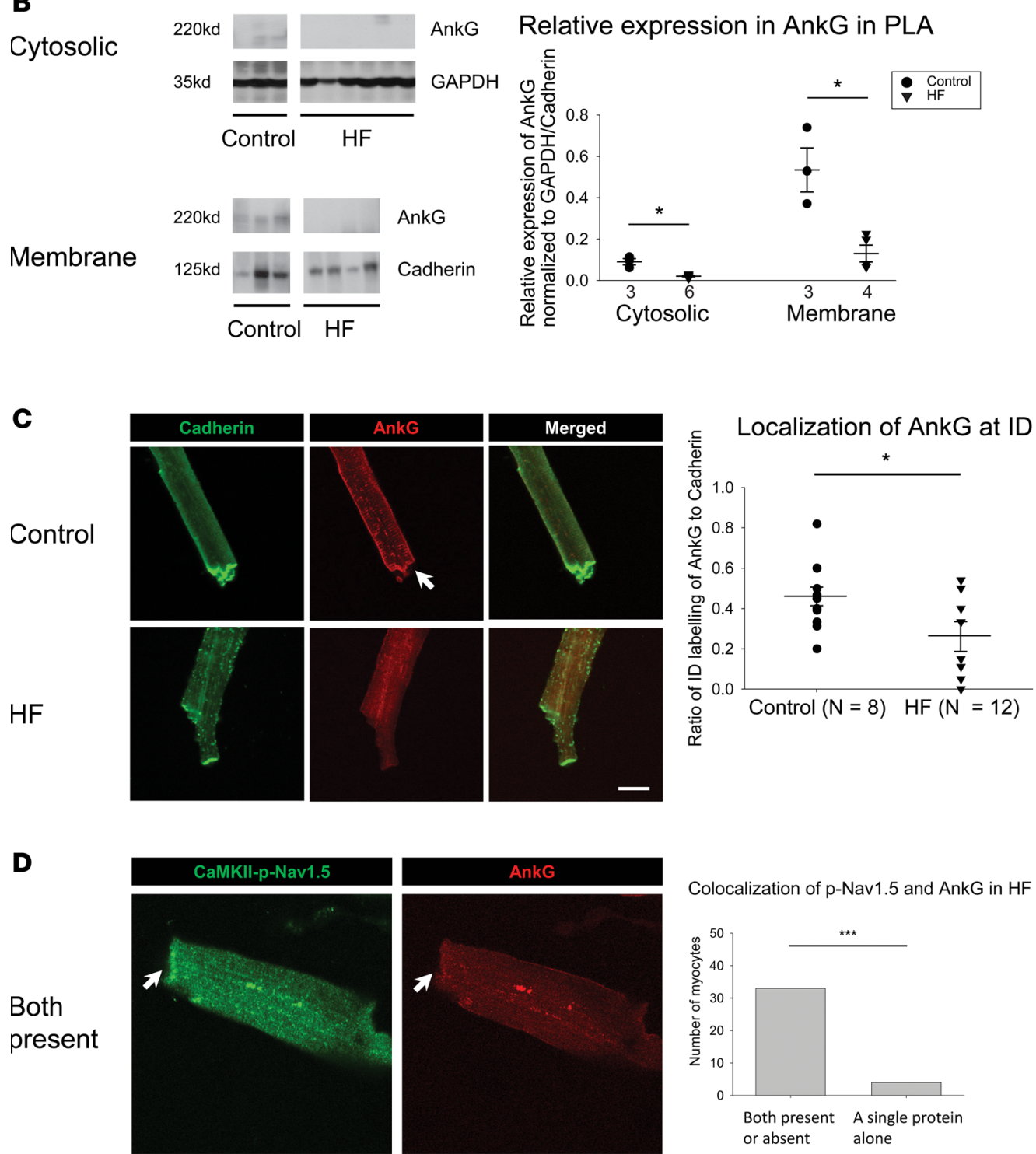

Colocalization of p-Nav1.5 and AnkG in HF

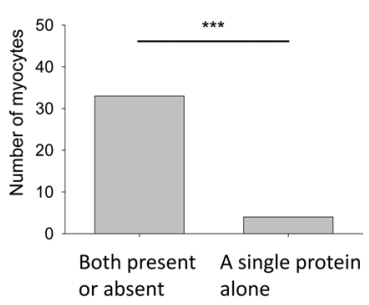

Both absent
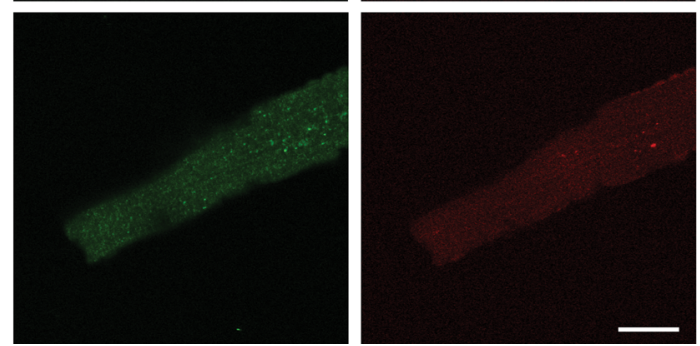
Figure 6. Decrease in expression and ID localization of AnkG is responsible for dropout of CaMKII-p-Na 1.5 (S571) in HF PLA. (A) In HF PLA, there was a dropout of CaMKII-p-Na 1.5 (S571) at certain ID (red arrow), where cadherin labeling was still intact at those ID (left). Quantification of myocytes with ID labeling of CaMKII-p-Nav1.5 (S571) in control and HF PLA (right). (B) Representative immunoblot and densitometric measurements of AnkG (normalized to GAPDH and cadherin) in cytosolic and membrane fractions, respectively, from control and HF PLA. (C) ID localization of AnkG (red) was reduced in isolated HF myocytes, while labeling of cadherin (green) seemed similar in control and HF myocytes. (D) Colocalization of CaMKII-p-Na 1.5 (S571) and AnkG at the ID (white arrows). Scale bar: $40 \mu \mathrm{m}$. Data are represented as mean \pm SEM. ${ }^{*} P<0.05,{ }^{* *} P<0.01,{ }^{* * *} P<0.001$. (A and B) Independent $t$ test. (C) Generalized linear model, logit link comparing rates if ID localization of AnkG. (D) $\chi^{2}$ test. HF, heart failure; PLA, posterior left atrium; ID, intercalated disc. See complete unedited blots in the supplemental material.

This patchy dropout of CaMKII-p-Na 1.5 (S571) at the ID led to an increase in spatial heterogeneity of CaMKII-p-Na 1.5 (S571) distribution in the HF PLA. As shown in Figure 7A, we quantified the ratio (numbers in yellow) of CaMKII-p-Na 1.5 (S571) (green) labeling at the ID against cadherin (red) labeling at the ID in a random sample of 9 images. The control images of the PLA showed a consistent CaMKII$\mathrm{p}-\mathrm{Na}_{\mathrm{v}} 1.5$ /cadherin ratio across all images (Figure 7A, left panel). However, in HF, some images showed a much higher ratio than others (Figure 7A, right panel). Thus, there was a significant increase in the coefficient of variation of CaMKII-p-Na 1.5 (S571)/cadherin ratio, compared with control PLA (Figure 7C). Interestingly, there was no significant difference in the coefficient of variation of CaMKII-p-Na 1.5 (S571)/cadherin ratio between control vs. HF LAA (Supplemental Figure 1B). We also assessed the spatial distribution of native $\mathrm{Na}_{\mathrm{v}} 1.5$. While the spatial distribution of $\mathrm{Na}_{\mathrm{v}} 1.5$ was more heterogeneous in the HF PLA compared with control, the increase in heterogeneity was less pronounced than noted with CaMKIIp-Na 1.5 (S571) (Figure 7, B and C).

Conduction changes in $\mathrm{HF}$ atrium correspond to the preferential increase in expression and enhanced spatial heterogeneity of Ox-CaMKII and CaMKII-p-Na 1.5 (S571) in the PLA

Previous reports have shown that $\mathrm{HF}$ contributes to the formation of AF substrate by promoting inhomogeneous conduction in the atrium $(23,28)$. However, it is not known whether there are regional differences in conduction slowing in the HF atria and whether conduction changes in the PLA are different from those elsewhere in the left atrium. Since OS appears to have a regional predilection for the HF PLA, resulting in a unique spatial distribution pattern of Ox-CaMKII and CaMKII-p-Na 1.5 (S571) at both the intra-cellular and inter-cellular level, we hypothesized that the conduction slowing and inhomogeneity previously described in the HF atrium occur preferentially in the HF PLA compared with the rest of the left atrium. We therefore performed high-density electrophysiological mapping in the left atrium at baseline and after the induction of HF, and we compared conduction characteristics between the PLA and the LAA. As shown in Figure 8, ventricular tachypacing-induced (VTP-induced) HF led to a decrease in CV during pacing at BCL of 400 and $300 \mathrm{~ms}$ in the PLA (Figure 8A). In the LAA, there was no significant change in $\mathrm{CV}$ with $\mathrm{HF}$ (Figure 8B). In addition to a slowing in $\mathrm{CV}$, there was also a marked increase in conduction inhomogeneity (CI) during sinus rhythm and during pacing at $300 \mathrm{~ms}$ in the PLA after 3 weeks of VTP (Figure 8C). However, there was no significant change in CI in LAA with VTP (Figure 8D).

The preferential decrease in CV seen in the PLA corresponds to the preferential increase in Ox-CaMKII and CaMKII-p-Na 1.5 (S571) levels noted in the PLA. Similarly, the increase in CI corresponds to the increased heterogeneity of spatial distribution of Ox-CaMKII and CaMKII-p-Na 1.5 (S571) in the HF PLA.

\section{Addition of slow conduction to existing fibrotic substrate enhances vulnerable substrate for AF in HF - insights from computation modeling}

As mentioned earlier, fibrosis is thought to be a key component of AF substrate formation in HF. The current study suggests that, in addition to fibrosis, OS-induced slowing of conduction via an increase in the level of CaMKII-p-Na 1.5 (S571) may also contribute to substrate for reentry in the HF PLA. To investigate whether slowing of conduction (e.g., by an increase in CaMKII-p-Na 1.5 [S571]) in the presence of existing fibrosis can exacerbate substrate for $\mathrm{AF}$ in the HF atrium, we performed a number of computer simulations in a 2-dimensional (2-D) atrial cell model, where vulnerability to wavebreak and reentry - and resulting sustained AF - was assessed in the presence of (a) a number of fibrotic conditions alone (Figure 9A,Supplemental Figure 8, and Supplemental Videos 1 and 4-6) and (b) regions of slow conduction that were juxtaposed to existing regions of fibrosis, either in a concentrated (Figure 9B and Supplemental Video 2) or scattered configuration (Figure 9C and Supplemental Video 3). As shown in Supplemental Figure 8, B and C, fibrosis alone was able to reliably cause sustained reentry only when a single extra stimulus was delivered close to 2 regions of fibrosis separated by a narrow isthmus of normal conduction. However, the addition of 
A $\quad$ Control
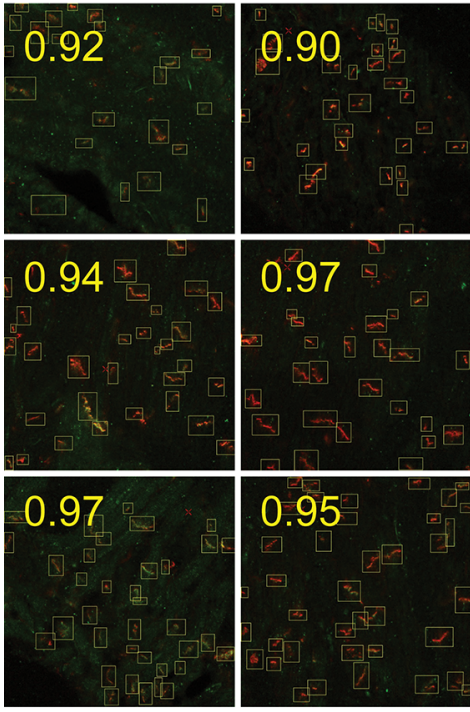

B
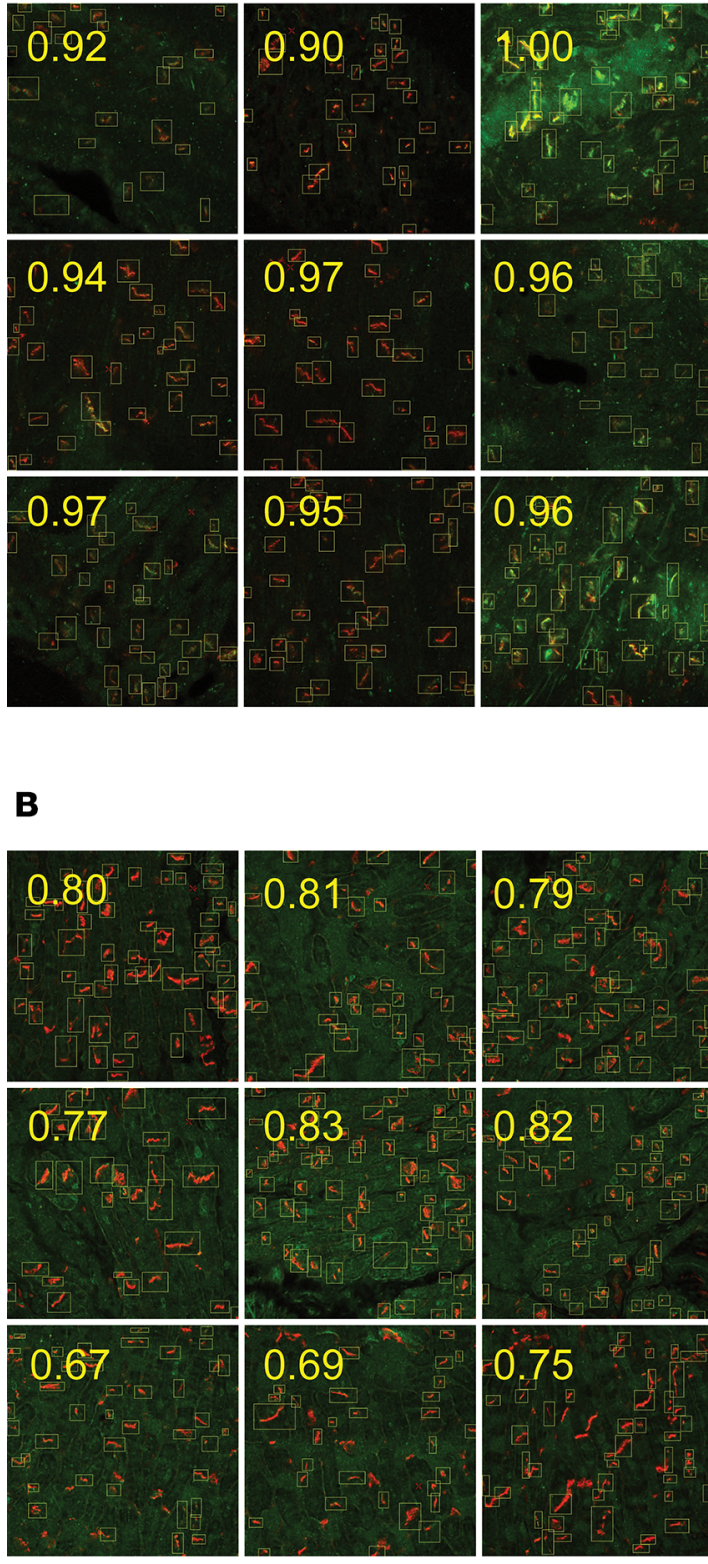

HF

PLA

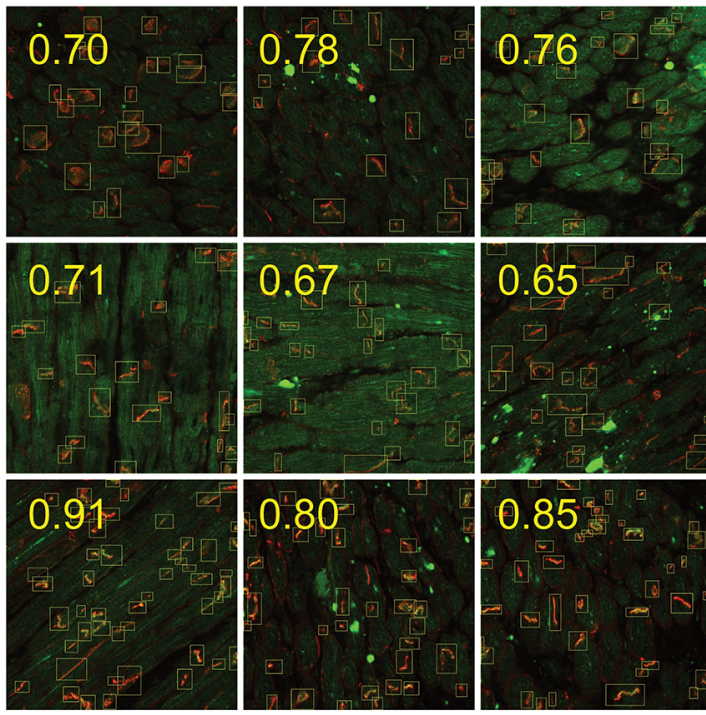

CaMKII-p-Na 1.5 Cadherin
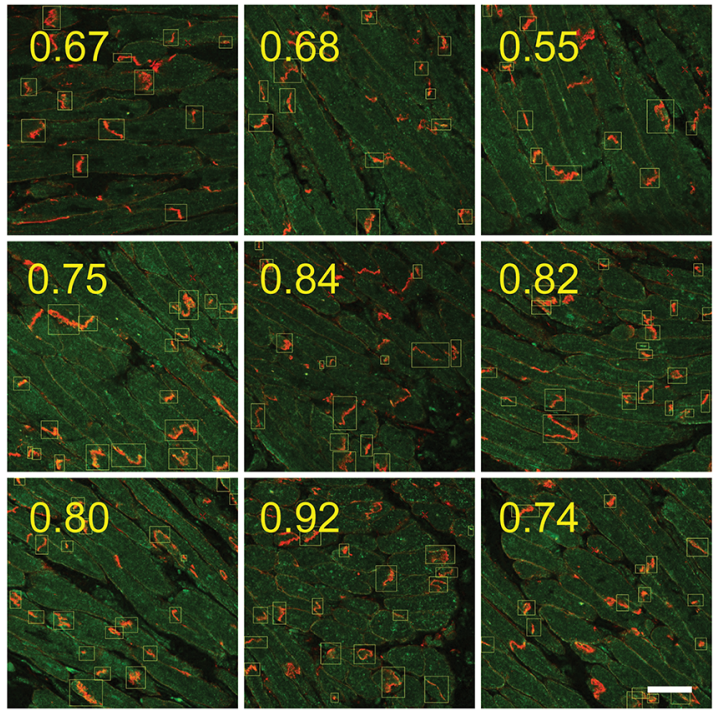

$\mathrm{Na}_{\mathrm{v}} 1.5$ Cadherin

C Heterogeneous distribution of p-Nav1.5 and Nav1.5 at IDs in PLA $\bullet \stackrel{\mathrm{HF}}{\text { Control }}$

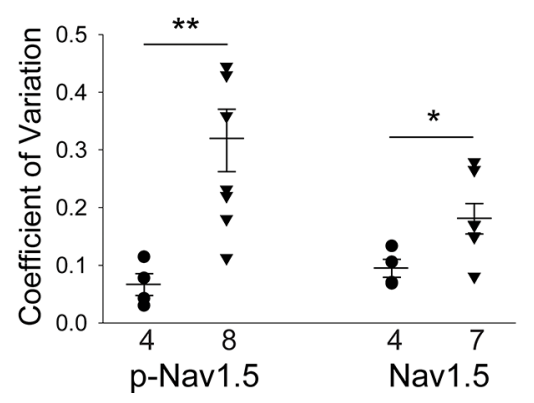

Figure 7. Increase in spatial heterogeneity of distribution of CaMKII-p-Na 1.5 (S571) in HF PLA. (A and B) More heterogeneous distribution of CaMKII-p-Nav1.5 (S571) (A) and $\mathrm{Na}_{\mathrm{v}} 1.5$ (B) in HF PLA, compared with control PLA in random nine $40 \times$ confocal images. Each number (yellow) represented the ratio of labeling frequency of CaMKII-p-Na 1.5 (S571) (A, green) and $\mathrm{Na}_{v} 1.5$ (B, green) against cadherin (red) at ID. Scale bar: $40 \mu \mathrm{m}$. (C) Comparison of spatial distribution of CaMKII-p- $\mathrm{Na}_{v} 1.5$ (S571) and $\mathrm{Na}_{v} 1.5$ in control and HF PLA. Note that CaMKII-p- $\mathrm{Na}_{v} 1.5$ (S571) was more heterogeneously distributed than $\mathrm{Na}_{v} 1.5$ in HF PLA. Data are represented as mean \pm SEM. ${ }^{*} P<0.05,{ }^{*} P<0.01$. Independent $t$ tests. HF, heart failure; PLA, posterior left atrium; ID, intercalated disc. 
A Conduction velocity in PLA

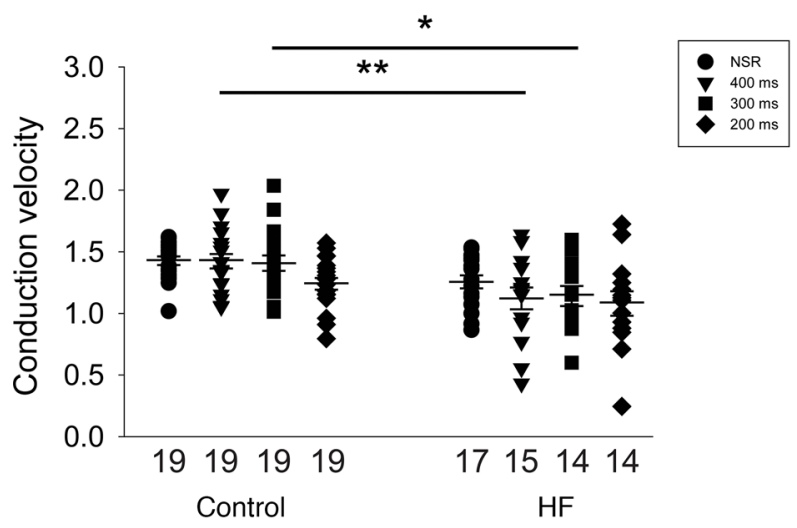

C Conduction inhomogeneity in PLA

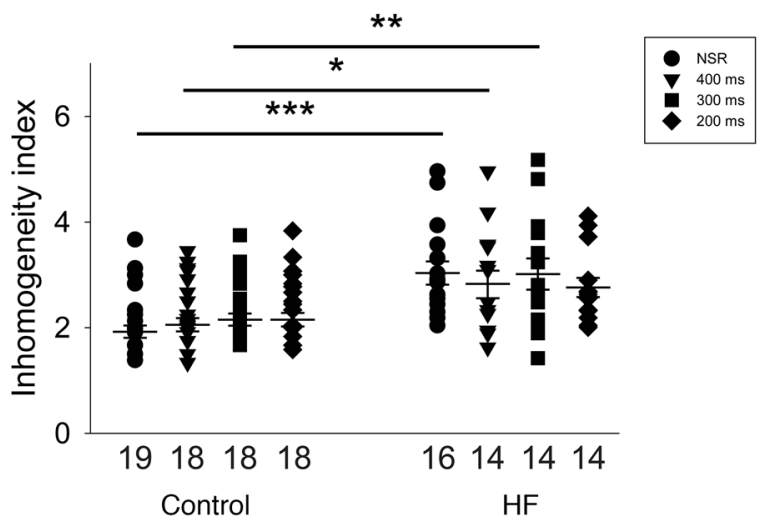

$\mathbf{E}$

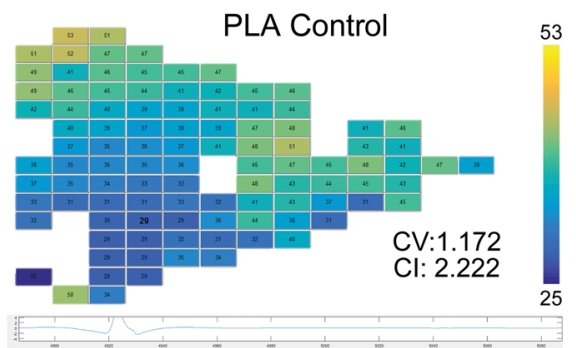

B Conduction velocity in LAA

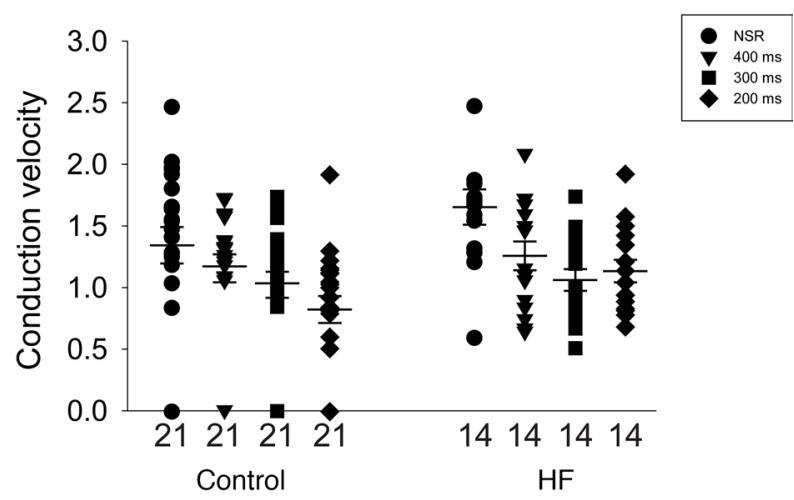

D Conduction inhomogeneity in LAA

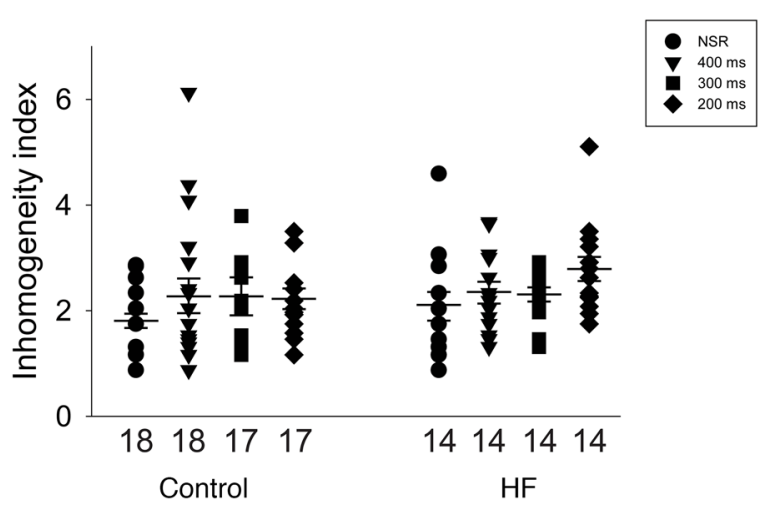

$\mathbf{F}$

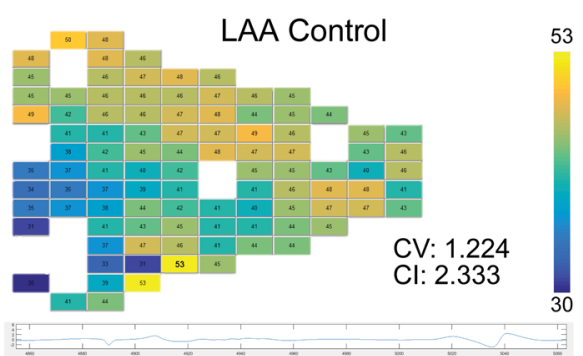

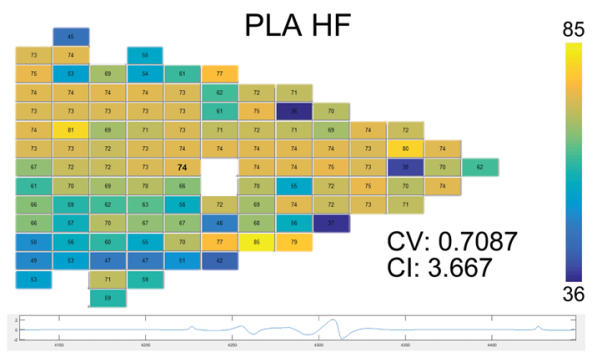

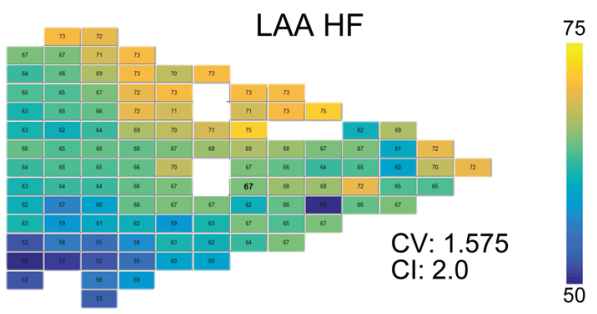

Figure 8. Change in conduction velocity and conduction inhomogeneity in PLA and LAA by ventricular tachypacing induced HF. (A and B) Conduction velocity was decreased at $400 \mathrm{~ms}$ and $300 \mathrm{~ms}$ cycle length in PLA after 3 weeks of ventricular tachypacing, but not in LAA. (C and D) Conduction inhomogeneity was increased at NSR, 400 ms, and 300 ms cycle length in PLA after 3 weeks of ventricular tachypacing, but not in LAA. (E and F) Representative examples of activation maps in the PLA and LAA, respectively. Data are represented as mean $\pm \mathrm{SEM} .{ }^{*} P<0.05,{ }^{* *} P<0.01,{ }^{* * *} P<0.001$. Repeated measures 2 -way ANOVA with Bonferroni adjusted within cycle comparisons. HF, heart failure; PLA, posterior left atrium; LAA, left atrial appendage. 


\section{A Simulation 1}
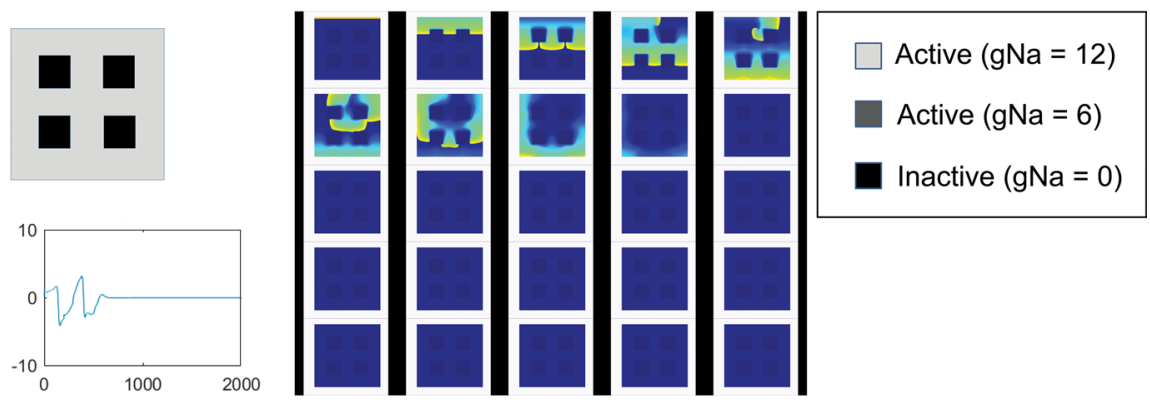

B

Simulation 2
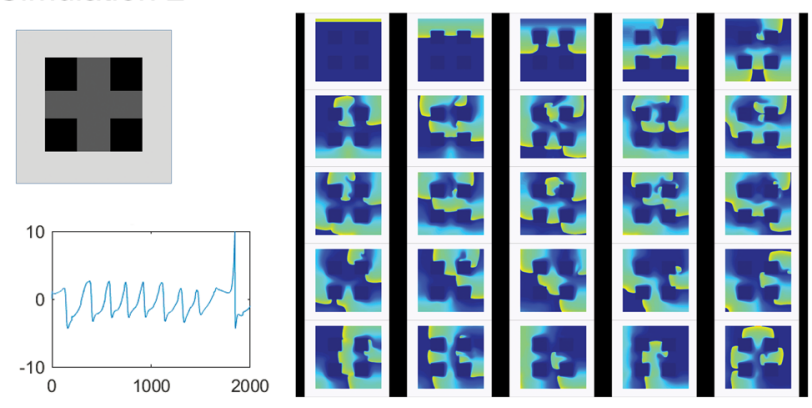

Figure 9. Mathematical simulation of action potential propagation in 2-D atrial tissue. Simulations were performed on a $200 \times$ 200 grid equivalent to a $5 \times 5 \mathrm{~mm}$ sheet of tissue. Dense fibrotic region was modeled as inactive and nonconducting areas ( $\mathrm{gNa}$ $=0$ ). Regions were assigned either normal ( $g \mathrm{Na}=12$ ) or reduced $(\mathrm{gNa}=6$ ) sodium conductance. Arrhythmia induction was performed in the presence of fibrosis alone (A) or reduced gNa regions juxtaposed to regions of fibrosis, either in a concentrated (B) or scattered configuration (C). In each condition, 25 screen shots of activation movie were taken every $75 \mathrm{~ms}$. Sustained reentry could be induced in the setting of condition $\mathbf{B}$ and $\mathbf{C}$, but not condition $\mathbf{A}$.

\section{Simulation 3}
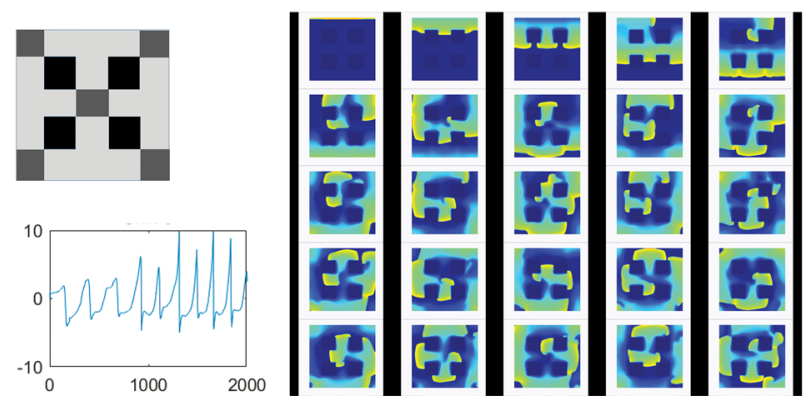

even small regions of slow conduction ( $50 \%$ of normal conduction) in and around regions of widely spaced, homogeneously distributed fibrosis - which would ordinarily not allow for the emergence of reentry - led to the initiation of wavebreak and sustained reentry (Figure 9, B and C). Furthermore, AF in the setting of the combination of fibrosis and patchy, slow conduction was significantly more disorganized than that noted in the setting of fibrosis alone (See pseudo ECGs in Figure 9, B and C, vs. Supplemental Figure 8, B and C).

\section{Discussion}

The findings of this study indicate that, in HF, OS creates a unique substrate in the PLA for both triggered activity and reentry (Figure 10). Specifically, our study demonstrates that there is increased OS in the HF left atrium, with the PLA demonstrating a preferential increase in OS compared with the rest of the left atrium. This increase in OS is accompanied by an increase in CaMKII phosphorylation of RyR2 and $\mathrm{Na}_{\mathrm{v}} 1.5$, likely secondary to an increase in expression of Ox-CaMKII. The increased CaMKII-phosphorylated RyR2 in the PLA appears to significantly enhance the propensity of HF PLA myocytes to initiate TCWs, which are highly sensitive to ROS inhibition. The increase in CaMKII-phosphorylated $\mathrm{Na}_{\mathrm{v}} 1.5$ in the HF PLA appears to create substrate for reentry by the following mechanisms: (a) unlike native $\mathrm{Na}_{\mathrm{v}} 1.5$, CaMKII-phosphorylated $\mathrm{Na}_{\mathrm{v}} 1.5$ is largely confined to a single compartment in the myocyte cell membrane (ID), which likely contributes to slowing of conduction in the HF PLA by leading to a decrease in peak $I_{\mathrm{Na}}$; (b) there is patchy dropout of CaMKII-phosphorylated $\mathrm{Na}_{\mathrm{v}} 1.5$ at the ID in HF (owing at least in part to a 

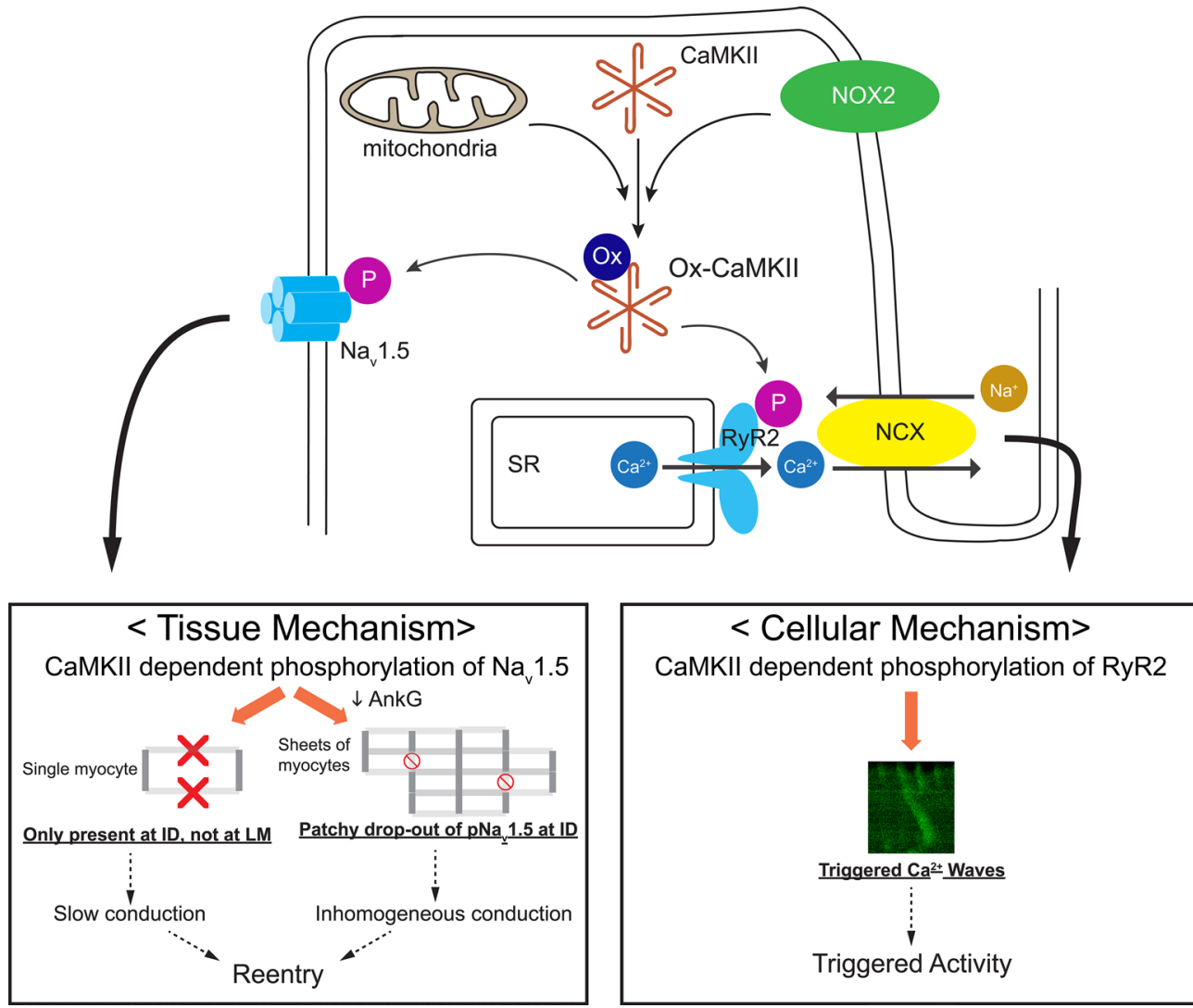

Figure 10. Schematic illustration of potential mechanisms by which 05 leads to substrate for triggered activity and reentry in the HF PLA. Increase in ROS generation by NADPH oxidase (NOX2) and mitochondrial ROS results in enhanced oxidation of CaMKII in the HF PLA. Ox-CaMKII hyperphosphorylates downstream target molecules, including RyR2 and $\mathrm{Na}_{v} 1.5$. At the cellular level, CaMKII-dependent phosphorylation of RyR2 leads to emergence of triggered $\mathrm{Ca}^{2+}$ waves, which predispose to afterdepolarizations (triggered activity). At the tissue level, CaMKII-dependent phosphorylation of $\mathrm{Na}_{v} 1.5$ results in selective trafficking of the channel protein to the ID, with little or no trafficking to the lateral membrane (LM). An increase in the level of CaMKII-p-Na 1.5 (S571) would therefore be expected to decrease peak $I_{\mathrm{Na}}$ and lead to conduction slowing. Furthermore, a decrease in AnkG levels in HF leads to defective forward trafficking of CaMKII-p-Na 1.5 (S571) to the ID; this results in a patchy dropout of CaMKII-p-Na 1.5 (S571) and, therefore, in increased heterogeneity of expression of CaMKII-p- $\mathrm{Na}_{v} 1.5$ (S571). The increased heterogeneity of expression of CaMKII-p-Na 1.5 (S571) promotes inhomogeneous conduction in the HF PLA. The conduction slowing and increased inhomogeneity of conduction caused by altered expression patterns of CaMKII-p-Nav 1.5 (S571) leads to substrate for reentry. HF, heart failure; PLA, posterior left atrium; ID, intercalated disc.

decrease in the level of its binding partner, AnkG). As a result, the spatial distribution of CaMKII-phosphorylated $\mathrm{Na}_{\mathrm{v}} 1.5$ is significantly more heterogeneous in HF compared with control PLA, corresponding closely to the preferential increase in conduction heterogeneity seen in the HF PLA compared with the rest of the left atrium. Computational modeling supported the notion that slowing of conduction in and around regions of atrial fibrosis in the HF atrium - as may be expected to be the case with increased expression and enhanced heterogeneity of spatial distribution of Ox-CaMKII and its downstream signaling partner CaMKII-phosphorylated $\mathrm{Na}_{v} 1.5$ - creates conditions that are more conducive to the emergence of sustained reentry and AF than the presence of fibrosis alone (i.e., fibrosis in the absence of neighboring regions of slow conduction).

Potential role of OS in creating electrical and structural remodeling in the intact PLA and in promoting a vulnerable substrate for $A F$. While it has been known for several years that ectopic foci originate in the PVs in most patients with $\mathrm{AF}$, the structural, electrophysiological, and molecular characteristics of this region that make it fertile for the emergence of these reentrant/triggered foci are just beginning to be understood. It is known that the PLA has a unique pattern of myocyte fiber orientation, which is thought to predispose to reentry (37). The PLA is also known to have peculiar electrophysiological characteristics, including longer refractory periods and significantly slower conduction than the rest of the atrium (38). 
Differences in refractory periods between the PLA/PVs and the adjoining left atrium are thought to be, at least in part, secondary to relative differences in ionic current properties between the PVs and the left atrium, with PV inward-rectifier currents being smaller and delayed-rectifier currents being larger in the PVs than in the left atrium $(39,40)$. In recent years, our laboratory and others have shown that the PVs/ PLA also demonstrate greater autonomic innervation than the rest of the left and right atriums, making this region more sensitive to autonomic manipulation - and therefore the emergence of reentry - than the rest of the atria (41-43). These baseline structural and electrophysiological differences between the PLA and the rest of the atrium appear to be exacerbated in the setting of the atrial remodeling that occurs with AF (44-46).

Unfortunately, the molecular mechanisms and signaling pathways that lead to electrical and structural remodeling in the PLA — and therefore to AF — are poorly understood. Studies in recent years have implicated OS as a likely mechanism in the creation of a vulnerable AF substrate. Mihm et al. demonstrated significant oxidative damage in atrial appendages of AF patients undergoing the Maze procedure (47). Carnes et al. showed that dogs with sustained AF had increased protein nitration, suggesting enhanced OS $(48,49)$. Kim et al. showed that NOX2 was a major source of atrial ROS in patients with AF (13). More recently, Reilly et al. showed that atrial sources of ROS vary with duration and substrate of AF, with NOX2 being elevated early in AF (e.g., postoperative AF) and with mitochondrial oxidases and uncoupled NOS being noted in long-standing AF (50). At the molecular level, OS creates substrate for AF by modifying key ion channels $(8-10)$ and activating profibrotic signaling $(11,12)$ in the atrium. Experimental evidence suggests that ROS-mediated disturbances of intracellular $\mathrm{Ca}^{2+}$ and $\mathrm{Na}^{+}$handling may result in electrical instability and create conditions conducive to the development of AF (51). In models of AF, ROS also increase cardiac RyR2 open probability, with the resulting sarcoplasmic reticulum (SR) $\mathrm{Ca}^{2+}$ leak thought to increase susceptibility to $\operatorname{AF}(52,53)$. This effect of ROS on RyR2 opening is thought to be at least partially mediated by CaMKII-dependent phosphorylation of RyR2 $(54,55)$.

In spite of these initial studies, the precise contribution of OS to electrophysiological remodeling in the intact atrium - and how this electrophysiological remodeling predisposes to reentry and triggered activity - is not well understood, nor is it known if OS is specifically involved in the genesis of AF in the PVs/PLA, whether or not structural heart disease is present. Since AF in the setting of HF is currently less amenable to ablation or antiarrhythmic drugs, a greater understanding of the AF disease state in the HF atrium - and how OS may be contributing to this disease state - would be crucial for the development of new, mechanism guided therapies for AF. To our knowledge, this is the first study that has systematically examined the role of OS in creation of a vulnerable substrate for AF in the HF PLA. Our results demonstrate a preferential increase in OS in the HF PLA, as compared with the rest of the left atrium. This increase in OS appears to cause both a general increase in protein oxidation in the PLA, as well as an increase in oxidation of CaMKII, an important kinase thought to be involved in electrophysiological signaling in the atrium. This preferential increase in oxidation in the PLA appears to contribute both to substrate for triggered activity — by increasing the propensity of PLA myocytes to harbor TCWs in response to rapid atrial rates - and to substrate for reentry, by leading to inhomogeneous conduction in the atrium via an increase in CaMKII phosphorylation of $\mathrm{Na}_{\mathrm{v}} 1.5$. Furthermore, to the best of our knowledge, this is the first demonstration of regional differences in oxidation in the intact $\mathrm{HF}$ atrium, especially in the context of how OS may be creating a vulnerable substrate for AF. In addition to having important mechanistic implications for the genesis of the AF disease state, these findings may also have important therapeutic implications for patients with AF (see below).

Our study also indicates that both NOX2 and mitochondrial ROS are preferentially elevated in the HF atrium, with both sources of OS appearing to contribute to the generation of TCWs. While the relative contribution of each source of OS to electrical and structural remodeling in the HF atrium is not clear, recent studies indicate the presence of significant cross-talk between these enzymatic sources of ROS, with Nox2 stimulating mitochondrial ROS by activating reverse electron transfer and with mitochondrial ATP-sensitive potassium channel-mediated (mitoK ${ }^{+}{ }_{\text {ATP }}$-mediated) mitochondrial $\mathrm{O}_{2-}$ stimulating cytoplasmic Nox 2 in endothelial cells $(56,57)$. This cross-talk may magnify the role of both sources of OS in the generation of triggered activity and reentry in the HF atrium.

Role of CaMKII in mediating OS-induced electrical remodeling in the PLA. CaMKII is a multifunctional serine-threonine protein kinase that is abundantly expressed in various tissues, with CaMKII $\delta$ being the predominant isoform in the heart. CaMKII may be activated or inactivated by posttranslational modifications, 
but posttranslational modifications to the CaMKII regulatory domain allow CaMKII to become persistently active under the influence of upstream signals that increase intracellular $\mathrm{Ca}^{2+}$ and $\mathrm{ROS}$ (58). CaMKII acts on a variety of downstream targets in cardiomyocytes by phosphorylating key targets, including voltage-gated ion channels (L-type $\mathrm{Ca}^{2+}$ channels, $\mathrm{K}^{+}$channels, and $\mathrm{Na}^{+}$channels) and $\mathrm{E}-\mathrm{C}$ coupling proteins (RyR2 and phospholamban) $(58,59)$. The net effect of CaMKII phosphorylation on these targets, by altering cellular excitability and by creating conditions conducive for afterdepolarizations, is to promote proarrhythmic conditions in myocytes. While most studies that examined the role of CaMKII on ion channels and E-C coupling have done so in the ventricle, at least a few studies have examined the role of CaMKII in the electrophysiology of isolated atrial myocytes. These suggest that CaMKII may be affecting atrial repolarization via effects on inward rectifying $\mathrm{K}$ current $\left(I_{\mathrm{K} 1}\right)$ and transient outward current $\left(I_{\text {to }}\right)(60,61)$. As in the ventricle, there is also evidence in the atrium that CaMKII affects - and is affected by - late $I_{\mathrm{Na}}$, thereby increasing substrate for afterdepolarizations $(62,63)$. Another mechanism by which CaMKII may increase substrate for triggered activity is via its effects on RyR2 phosphorylation, specifically at the serine 2814 site (55). Although these studies suggest that CaMKII may contribute to a vulnerable substrate for AF, there is a paucity of studies that examined how CaMKII contribute to AF substrate in the intact atrium. A key mechanism that is thought to underlie AF substrate formation, especially in the setting of $\mathrm{HF}$, is slow and inhomogeneous conduction (23). There is some evidence that increased CaMKII activity by enhanced oxidation may lead to slow conduction in the post-myocardial infarction ventricle, with computational modeling suggesting that at least part of this slowing may result from altered $\mathrm{Na}^{+}$channel kinetics (32). Nonetheless, the precise electrophysiological mechanisms by which increased CaMKII may be slowing conduction in the ventricle - and whether a similar mechanism may be operative in the HF atrium - has not hitherto been studied.

In this study, we have examined the effect of CaMKII signaling on triggered activity and on intact atrial conduction - in a clinically relevant large-animal model of AF. The enhanced CaMKII signaling in this model appears to be caused in large part by increased OS. Once activated, CaMKII appears to affect atrial electrophysiology, possibly via its phosphorylation of RyR2 and $\mathrm{Na}_{\mathrm{v}} 1.5$. Enhanced CaMKII-p-RyR2 (S2814) has been shown in several studies to increase afterdepolarizations in both ventricular and atrial myocytes, by increasing $\mathrm{Ca}^{2+}$ leak from RyR2 receptors. Voigt et al. showed that CaMKII-p-RyR2 (S2814) leads to an increased propensity for spontaneous $\mathrm{Ca}^{2+}$ release $\left(\mathrm{Ca}^{2+}\right.$ waves $)$ - and consequent delayed afterdepolarizations - in atrial myocytes from patients with chronic AF (16). Very recently, we have shown that atrial myocytes demonstrate a propensity for $\mathrm{Ca}^{2+}$ waves that occur in the setting of rapid heart rates (17). These TCWs occur during atrial systole and cause membrane depolarizations that are conducive to the genesis of early-afterdepolarizations (30). Atrial myocytes appear prone to these waves since they have a poorly developed T-tubular system - at least in some species - so that $\mathrm{Ca}^{2+}$ signaling occurs mostly on the cell boundary. This paucity of T-tubules appears to be especially pronounced in canine atrial myocytes, as we have reported recently (64). In our computational study, we found that CaMKII phosphorylation of RyR2, which is induced by increased ROS activity, favors $\mathrm{Ca}^{2+}$ wave propagation by reducing the activation threshold for $\mathrm{Ca}^{2+}$ sparks. This is due to the increased open probability of CaMKII-phosphorylated RyR2. Thus, the signaling architecture of atrial myocytes makes them particularly prone to abnormal $\mathrm{Ca}^{2+} \mathrm{cycling}$ activity caused by a disruption of $\mathrm{Ca}^{2+}$ signaling. Notably, recent reports indicate the presence of voluminous axial tubules - at least in mouse and human atria - with extensive junctions to the SR that include RyR2 clusters (65). Even if T-tubules are somewhat well developed in some species, T-tubule breakdown would not be unexpected in the setting of HF, based on our prior work in the HF ventricle where significant T-tubule breakdown occurs with advancing HF (66). Future studies are needed to determine whether a relationship exists between TCWs and T-tubule architecture in these other species.

The incidence of TCWs increases significantly in $\mathrm{HF}$ myocytes, with $\mathrm{Ca}^{2+}$ waves occurring at significantly slower cycle lengths than in normal myocytes. These TCWs are highly sensitive to CaMKII blockade, implicating CaMKII-dependent RyR2 phosphorylation as a potential mechanism underlying these TCWs. In the current study, we have demonstrated that these TCWs are markedly sensitive to ROS blockade, especially in myocytes from the PLA. Taken together, these data strongly suggest that OS creates substrate for TCWs in the HF PLA and does so, at least in part, by increasing CaMKII signaling in the atrium.

Importantly, the PLA has been shown to harbor substrate for not only triggered activity, but also reentry (38), with most rapidly firing PV foci thought to be reentrant in nature. Furthermore, since HF leads to both electrical and structural remodeling in the atrium, reentry may be a key electrophysiological mechanism involved in the maintenance of the AF disease in HF. We therefore examined whether OS affects 
conduction characteristics in the intact atrium via CaMKII-p-Na 1.5 (S571). Surprisingly, we discovered that, unlike native $\mathrm{Na}_{\mathrm{v}} 1.5$, CaMKII-p- $\mathrm{Na}_{\mathrm{v}} 1.5$ (S571) is almost entirely absent at the lateral membrane in atrial myocytes. Recent work suggests that CaMKII may be involved in the forward trafficking of $\mathrm{Na}_{\mathrm{v}} 1.5$ to the cell membrane, in good part due to its interactions with cytoskeletal adaptor proteins such as AnkG and $\beta I V$-spectrin $(15,26,36)$. Several reports in recent years demonstrate the presence of different pools of ion channels in the cardiac cell membrane. This compartmentalization of ion channel proteins is thought to occur in large part due to binding to trafficking proteins such as the dystrophin-syntrophin multiprotein complex, SAP97 and AnkG $(67,68)$. Of these, the dystrophin-syntrophin macromolecular complex appears to target $\mathrm{Na}_{\mathrm{v}} 1.5$ to the lateral membrane region of the cardiomyocyte (35). Other reports demonstrate interaction at the ID between gap junctions, desmosomes, and the $\mathrm{Na}_{\mathrm{v}} 1.5$ channel complex through the interactions of $\mathrm{Cx} 43$, pkp2, and AnkG $(34,69)$. The selective compartmentalization of CaMKII-p$\mathrm{Na}_{\mathrm{v}} 1.5$ (S571) to the ID - noted in both control and HF atria in our experiments - is likely due to the previously demonstrated interaction between CaMKII, $\mathrm{Na}_{\mathrm{v}} 1.5, \mathrm{AnkG}$, and $\beta I V$-spectrin, which have been shown to coexist within a large macromolecular complex (36). This finding has significant implications for the conduction slowing and inhomogeneous conduction that is typically noted in the HF atrium, especially in the PLA. Indeed, as demonstrated by Shy et al., dropout of $\mathrm{Na}_{\mathrm{v}} 1.5$ at the lateral membrane results in approximately $40 \%$ decrease in peak $I_{\mathrm{Na}}$, which probably contributes to significant conduction slowing in the ventricle (35). In light of these findings, it is highly likely that the slow and inhomogeneous conduction that characterize AF substrate in HF may be at least partially mediated by an OS-induced increase in CaMKII-p-Na 1.5 (S571). The importance of AnkG in trafficking CaMKII-p-Na 1.5 (S571) to the ID is further underscored by the observation that, even though Ox-CaMKII activity increases in HF, CaMKII-p-Na 1.5 expression at the ID - the only compartment where it preferentially exists - decreases. Our data indicate that this patchy dropout of CaMKII-p- $\mathrm{Na}_{\mathrm{v}} 1.5$ is secondary to a significant decrease in AnkG in the HF atrium. Our computational modeling studies strongly support a role for preferential conduction slowing in the PLA — such as that resulting from decreased $\mathrm{Na}_{\mathrm{v}} 1.5$ availability owing to the above-mentioned mechanisms - for the creation of reentrant substrate in the atrium. It has long been known that fibrosis creates important structural barriers in the atrium that are necessary for the emergence of reentry $(20,23)$. Importantly, our computational studies suggest that fibrosis, while a key component of inhomogeneous conduction slowing in the atrium, may alone not always be sufficient for the creation of conditions conducive for reentry. It appears that - in the presence of interstitial fibrosis, which establishes inexcitable conduction barriers in both in the PLA and in the LAA — further conduction slowing by decreased availability of $I_{\mathrm{Na}}$ (e.g., due to an increase in CaMKII phosphorylated $\mathrm{Na}_{\mathrm{v}} 1.5$ ) may more readily create conditions conducive to reentry in the PLA than would be possible in the presence of fibrosis alone.

Implications for therapeutic targeting of AF in HF. Currently, success of both pharmacological and ablative therapies for AF in patients with HF is significantly below that of patients without structural heart disease (70). The low success of ablation in patients with structural heart disease or in patients with long-standing persistent $\mathrm{AF}$ is thought to be at least in part because AF ablation in its current form is largely anatomic, with PV isolation (the primary objective of ablation) being inadequate to sufficiently disrupt the key molecular mechanisms (e.g., OS) responsible for the genesis and maintenance of AF in HF. Recent attempts at ablating large amounts of atrial myocardium - by adding linear ablation in the atrium or ablating complex fractionated atrial electrograms - have not demonstrated a significant increase in ablation success in patients with long-standing persistent AF and/or structural heart disease (71). Furthermore, it is well known that excessive ablation in the atrium is associated with significant risks, including collateral damage to surrounding structures and a long-term risk of decreased atrial function (72). Due to these inherent limitations of ablation, future therapeutic strategies for patients with AF in HF should consider biological therapies that can selectively target OS and/or key signaling pathways involved in the creation of this substrate (e.g., CaMKII), without causing myocardial damage. Recent years have seen promising results with gene-based therapies that have targeted both electrical and structural remodeling in large-animal models of $\mathrm{AF}$, including an AF model of HF (73-77). The results of the current study suggest that targeted reduction of OS in the HF PLA with such a strategy may have a beneficial effect on both triggered and reentrant substrate for AF. Furthermore, since OS is also thought to contribute to the creation of atrial fibrosis, targeted inhibition of OS with a gene-based therapy may also lead to the prevention of fibrosis in the longer term.

Study limitations and future directions. Our study did not investigate precisely why the PLA should generate more OS than the surrounding left atrium. A potential explanation may be differences in stretch 
between different atrial regions (during atrial systole), as well as excess OS generation due to the high concentration of sympathetic nerves in the PLA. Future studies are needed to look at the fundamental mechanisms that lead to greater OS generation in the PLA.

Another area of future investigation would be to link the increased heterogeneity of Ox-CaMKII distribution in HF PLA with downstream E-C coupling effectors. Since canine atria have little or no T-tubules and since there appears to be no gross disruption of RyR2 receptors in the canine HF atrium (unpublished data), higher-resolution imaging using techniques such as direct stochastic optical reconstruction microscopy (d-STORM) will be need to examine ultrastructural changes in RyR2 distribution and to correlate these changes with upstream CaMKII signaling. Related studies should also assess how more targeted disruption of ROS - using specific probes such as the NOX2 inhibitory peptide gp-91-tat - affect E-C coupling and TCW generation in HF atrial myocytes.

Since HF atria in our study clearly show both fibrosis and an increase in CaMKII-p-Na 1.5 , it is difficult to tease out the relative contribution of each mechanism to conduction changes seen in the atrium in HF. In the absence of a pure animal model of conduction slowing/inhomogeneity due to increase in CaMKII-p-Na 1.5 , we are currently unable to compare our mathematical predictions with actual mapping studies. Future studies are necessary to assess CaMKII-p-Na 1.5 expression/distribution at earlier time points during tachypacing-induced $\mathrm{HF}$; since these earlier time points would be expected to have less fibrosis, any conduction changes seen would be expected to be largely due to electrical remodeling (e.g., secondary to an increase in CaMKII-p-Na $\mathrm{N}_{\mathrm{v}} 1.5$ ).

\section{Methods}

Supplemental Methods are available online with this article.

Hound dogs used in this study weighed $25-35 \mathrm{Kg}$ and were aged $\geq 1$ year. Canine HF model was induced by right VTP (240 beats/min) for 3 weeks. Two groups of dogs were studied: HF $(n=33)$ and controls $(n=26)$. Animals were subjected to 1 or more of the following types of studies: (a) In vivo electrophysiological study, (b) studies in isolated myocytes for $\mathrm{Ca}^{2+}$ cycling and immunocytochemistry, or (c) tissue analysis (lucigenin chemiluminescence, carbonylation, immunoblotting, and IHC). Mathematical modeling of spatiotemporal distribution of $\mathrm{Ca}^{2+}$ in an atrial cell model was performed to better understand the connection between OS, CaMKII signaling, and TCW generation at different levels of ROS. (32, 33, $78,79)$. Two-dimensional mathematical simulation for an atrial action potential was performed to study influence of slow conduction on AF generation in the presence of fibrosis (80).

Statistics. Data are represented as mean \pm SEM. The number of samples (animals or isolated myocytes) for each experiment is given in each figure panel. Essentially, when control and HF animals were directly compared, independent 2-tailed $t$ tests were used; when comparisons were made within control and HF animals, 1- or 2-way ANOVA methods were used. The statistical analyses performed for each experiment are provided in each figure legend. An overview of the statistical analysis used is given below. Some caution should be used when making inferences pertaining to the mean, SEM, and $P$ values, as sample sizes are restrictive.

Comparison of tissue parameters (e.g., superoxide generation, immunoblot) between $\mathrm{HF}$ and control animals was performed using independent $t$ tests (2 tailed). Comparison of different sources of ROS in PLA and LAA was performed using 2-way ANOVA. Comparison of tissue parameters within regions (PLA vs. LAA) within HF or control animals was performed using independent $t$ tests (2-tailed).

The incidence of TCWs (and other $\mathrm{Ca}^{2+}$ cycling parameters) was compared between $\mathrm{HF}$ and control myocytes using independent $t$ tests (2-tailed). TCWs were compared in the presence of different interventions using an ANOVA. Comparison of conduction parameters (at different pacing cycle lengths) in control and HF dogs was performed using 2-way ANOVA. In instances where an ANOVA with main effects with multiple categories was statistically significant, post hoc pairwise $t$ tests were performed with a Bonferroni correction to adjust for multiple comparisons. Quantification of myocytes with LM labeling of $\mathrm{Na}_{\mathrm{v}} 1.5$ and CaMKII-p-Na 1.5 (S571) in control and HF myocytes were compared within group using a 2-way ANOVA. ID localization of AnkG was compared across control and HF myocytes using generalized linear models with a logit link to account for multiple observations and detection. Colocalization of CaMKII-p-Na 1.5 (S571) and AnkG at the ID was compared using a $\chi^{2}$ test. All statistical comparisons were run in SigmaPlot at a significance level of 0.05 .

Study approval. Hound dogs used in this study were maintained in accordance to the Guide for the Care and Use of Laboratory Animals published by the U.S. NIH (publication no. 85-23, revised 1996). The study was approved by the animal care and use committee (IACUC) of Northwestern University. 


\section{Author contributions}

SY and RA conceived and designed experiments and wrote the manuscript. SY conducted most of the experiments, acquired data, analyzed the data, and prepared figures. GA contributed to experimental design and $\mathrm{Ca}^{2+}$ imaging data analysis. PJM and TJH provided essential reagents. YS and JN conducted mathematical simulations. SB and TW conducted immunoblot analysis. GG contributed to conduction data analysis. MG contributed to immunohistochemical data analysis. SY, GA, YS, JN, PJM, TJH, TW, BPK, RP, JJG, JAW, and RA contributed to the Discussion section.

\section{Acknowledgments}

We would like to thank X. Wehrens at Baylor College of Medicine for providing a rabbit polyclonal antibody for CaMKII-p-RyR2 (S2814), Bassel Shanab for assistance in image analysis of IHC results, Wenwei Zhang for assistance with some of the protein chemistry experiments, and Mary Kwasny for assistance with biostatistical methods. This work was supported by the following grants: RA (NIH HL093490 and HL140061), Northwestern University Proof-of-concept Biomedical award, and Northwestern Memorial Foundation (Dixon award). JAW was supported by NIH HL119095. PJM was supported by NIH HL135754, HL134824, HL139348, HL135096, and HL114383. TJH was supported by NIH HL-135096, HL134824, and HL114893.

Address correspondence to: Rishi Arora, Northwestern University-Feinberg School of Medicine, 251 East Huron, Feinberg 8-503, Chicago, Illinois 60611, USA. Phone: 312.503.3217; Email: r-arora@northwestern.edu.

1. Benjamin EJ, Levy D, Vaziri SM, D'Agostino RB, Belanger AJ, Wolf PA. Independent risk factors for atrial fibrillation in a population-based cohort. The Framingham Heart Study. JAMA. 1994;271(11):840-844.

2. Gerstenfeld EP, et al. Predictors of success after selective pulmonary vein isolation of arrhythmogenic pulmonary veins for treatment of atrial fibrillation. Heart Rhythm. 2006;3(2):165-170.

3. Pappone C, et al. Atrio-esophageal fistula as a complication of percutaneous transcatheter ablation of atrial fibrillation. Circulation. 2004;109(22):2724-2726.

4. Verma A, et al. Efficacy of adjuvant anterior left atrial ablation during intracardiac echocardiography-guided pulmonary vein antrum isolation for atrial fibrillation. J Cardiovasc Electrophysiol. 2007;18(2):151-156.

5. Rodrigues AC, et al. Left atrial function after ablation for paroxysmal atrial fibrillation. Am J Cardiol. 2009;103(3):395-398

6. Aldhoon B, Melenovský V, Peichl P, Kautzner J. New insights into mechanisms of atrial fibrillation. Physiol Res. 2010;59(1):1-12.

7. Jeong EM, et al. Metabolic stress, reactive oxygen species, and arrhythmia. J Mol Cell Cardiol. 2012;52(2):454-463.

8. Hool LC. Reactive oxygen species in cardiac signalling: from mitochondria to plasma membrane ion channels. Clin Exp Pharmacol Physiol. 2006;33(1-2):146-151.

9. Zima AV, Blatter LA. Redox regulation of cardiac calcium channels and transporters. Cardiovasc Res. 2006;71(2):310-321.

10. Nediani C, Raimondi L, Borchi E, Cerbai E. Nitric oxide/reactive oxygen species generation and nitroso/redox imbalance in heart failure: from molecular mechanisms to therapeutic implications. Antioxid Redox Signal. 2011;14(2):289-331.

11. Lijnen PJ, van Pelt JF, Fagard RH. Stimulation of reactive oxygen species and collagen synthesis by angiotensin II in cardiac fibroblasts. Cardiovasc Ther. 2012;30(1):e1-e8.

12. Nabeebaccus A, Zhang M, Shah AM. NADPH oxidases and cardiac remodelling. Heart Fail Rev. 2011;16(1):5-12.

13. Kim YM, et al. A myocardial Nox2 containing NAD(P)H oxidase contributes to oxidative stress in human atrial fibrillation. Circ Res. 2005;97(7):629-636.

14. Purohit A, et al. Oxidized $\mathrm{Ca}(2+)$ /calmodulin-dependent protein kinase II triggers atrial fibrillation. Circulation. 2013;128(16):1748-1757.

15. Hund TJ, et al. A $\beta(\mathrm{IV})$-spectrin/CaMKII signaling complex is essential for membrane excitability in mice. J Clin Invest. 2010;120(10):3508-3519.

16. Voigt $\mathrm{N}$, et al. Enhanced sarcoplasmic reticulum $\mathrm{Ca}^{2+}$ leak and increased $\mathrm{Na}^{+}-\mathrm{Ca}^{2+}$ exchanger function underlie delayed afterdepolarizations in patients with chronic atrial fibrillation. Circulation. 2012;125(17):2059-2070.

17. Aistrup GL, et al. Triggered intracellular calcium waves in dog and human left atrial myocytes from normal and failing hearts. Cardiovasc Res. 2017;113(13):1688-1699.

18. Hohendanner F, et al. Inositol-1,4,5-trisphosphate induced Ca2+ release and excitation-contraction coupling in atrial myocytes from normal and failing hearts. J Physiol (Lond). 2015;593(6):1459-1477.

19. Wasserstrom JA, et al. Multiple defects in intracellular calcium cycling in whole failing rat heart. Circ Heart Fail. 2009;2(3):223-232

20. Andrade J, Khairy P, Dobrev D, Nattel S. The clinical profile and pathophysiology of atrial fibrillation: relationships among clinical features, epidemiology, and mechanisms. Circ Res. 2014;114(9):1453-1468.

21. Heijman J, Voigt N, Nattel S, Dobrev D. Cellular and molecular electrophysiology of atrial fibrillation initiation, maintenance, and progression. Circ Res. 2014;114(9):1483-1499.

22. Nattel S, Dobrev D. Electrophysiological and molecular mechanisms of paroxysmal atrial fibrillation. Nat Rev Cardiol. 2016;13(10):575-590.

23. Li D, Fareh S, Leung TK, Nattel S. Promotion of atrial fibrillation by heart failure in dogs: atrial remodeling of a different sort. Circulation. 1999;100(1):87-95. 
24. Li D, et al. Effects of experimental heart failure on atrial cellular and ionic electrophysiology. Circulation. 2000;101(22):2631-2638.

25. Poulet $\mathrm{C}$, et al. Late sodium current in human atrial cardiomyocytes from patients in sinus rhythm and atrial fibrillation. $P L o S$ One. 2015;10(6): $\mathrm{e} 0131432$.

26. Glynn P, et al. Voltage-gated sodium channel phosphorylation at Ser571 regulates late current, arrhythmia, and cardiac function in vivo. Circulation. 2015;132(7):567-577.

27. Hund TJ, et al. Role of activated CaMKII in abnormal calcium homeostasis and $\mathrm{I}(\mathrm{Na})$ remodeling after myocardial infarction insights from mathematical modeling. J Mol Cell Cardiol. 2008;45(3):420-428.

28. Schotten U, Verheule S, Kirchhof P, Goette A. Pathophysiological mechanisms of atrial fibrillation: a translational appraisal. Physiol Rev. 2011;91(1):265-325.

29. Heijman J, Voigt N, Ghezelbash S, Schirmer I, Dobrev D. Calcium Handling Abnormalities as a Target for Atrial Fibrillation Therapeutics: How Close to Clinical Implementation? J Cardiovasc Pharmacol. 2015;66(6):515-522.

30. Gussak G, Marszalec W, Shiferaw S, Wasserstrom JA. Electrophysiological effects of triggered calcium waves on the action potential in left atrial myocytes from normal and failing dog heart. Biophys J. 2017;112(3):235a.

31. Joseph LC, et al. Mitochondrial oxidative stress during cardiac lipid overload causes intracellular calcium leak and arrhythmia. Heart Rhythm. 2016;13(8):1699-1706.

32. Christensen MD, Dun W, Boyden PA, Anderson ME, Mohler PJ, Hund TJ. Oxidized calmodulin kinase II regulates conduction following myocardial infarction: a computational analysis. PLoS Comput Biol. 2009;5(12):e1000583.

33. Hashambhoy YL, Greenstein JL, Winslow RL. Role of CaMKII in RyR leak, EC coupling and action potential duration: a computational model. J Mol Cell Cardiol. 2010;49(4):617-624.

34. Shy D, Gillet L, Abriel H. Cardiac sodium channel NaV1.5 distribution in myocytes via interacting proteins: the multiple poo model. Biochim Biophys Acta. 2013;1833(4):886-894

35. Shy D, et al. PDZ domain-binding motif regulates cardiomyocyte compartment-specific NaV1.5 channel expression and function. Circulation. 2014;130(2):147-160.

36. Makara MA, et al. Ankyrin-G coordinates intercalated disc signaling platform to regulate cardiac excitability in vivo. Circ Res. 2014;115(11):929-938.

37. Hocini M, et al. Electrical conduction in canine pulmonary veins: electrophysiological and anatomic correlation. Circulation. 2002;105(20):2442-2448.

38. Arora R, et al. Arrhythmogenic substrate of the pulmonary veins assessed by high-resolution optical mapping. Circulation 2003;107(13):1816-1821.

39. Melnyk P, Ehrlich JR, Pourrier M, Villeneuve L, Cha TJ, Nattel S. Comparison of ion channel distribution and expression in cardiomyocytes of canine pulmonary veins versus left atrium. Cardiovasc Res. 2005;65(1):104-116.

40. Ehrlich JR, et al. Cellular electrophysiology of canine pulmonary vein cardiomyocytes: action potential and ionic current properties. J Physiol (Lond). 2003;551(pt 3):801-813.

41. Arora R, et al. Unique autonomic profile of the pulmonary veins and posterior left atrium. J Am Coll Cardiol. 2007;49(12):1340-1348

42. Arora R, et al. Neural substrate for atrial fibrillation: implications for targeted parasympathetic blockade in the posterior left atrium. Am J Physiol Heart Circ Physiol. 2008;294(1):H134-H144.

43. Ng J, et al. Autonomic remodeling in the left atrium and pulmonary veins in heart failure: creation of a dynamic substrate for atrial fibrillation. Circ Arrhythm Electrophysiol. 2011;4(3):388-396.

44. Zheng S, et al. Autonomic neural remodeling of the pulmonary vein-left atrium junction in a prolonged right atrial pacing canine model. Pacing Clin Electrophysiol. 2014;37(6):745-750.

45. Teh AW, et al. Electroanatomic properties of the pulmonary veins: slowed conduction, low voltage and altered refractoriness in AF patients. J Cardiovasc Electrophysiol. 2011;22(10):1083-1091.

46. Colman MA, Varela M, Hancox JC, Zhang H, Aslanidi OV. Evolution and pharmacological modulation of the arrhythmogenic wave dynamics in canine pulmonary vein model. Europace. 2014;16(3):416-423.

47. Mihm MJ, et al. Impaired myofibrillar energetics and oxidative injury during human atrial fibrillation. Circulation. 2001;104(2):174-180.

48. Carnes CA, et al. Atrial glutathione content, calcium current, and contractility. J Biol Chem. 2007;282(38):28063-28073.

49. Carnes CA, et al. Ascorbate attenuates atrial pacing-induced peroxynitrite formation and electrical remodeling and decreases the incidence of postoperative atrial fibrillation. Circ Res. 2001;89(6):E32-E38.

50. Reilly SN, et al. Atrial sources of reactive oxygen species vary with the duration and substrate of atrial fibrillation: implications for the antiarrhythmic effect of statins. Circulation. 2011;124(10):1107-1117.

51. Wagner S, Rokita AG, Anderson ME, Maier LS. Redox regulation of sodium and calcium handling. Antioxid Redox Signal. 2013;18(9):1063-1077.

52. Xie W, et al. Mitochondrial oxidative stress promotes atrial fibrillation. Sci Rep. 2015;5:11427.

53. Li N, et al. Ryanodine receptor-mediated calcium leak drives progressive development of an atrial fibrillation substrate in a transgenic mouse model. Circulation. 2014;129(12):1276-1285.

54. Erickson JR, et al. A dynamic pathway for calcium-independent activation of CaMKII by methionine oxidation. Cell. 2008;133(3):462-474.

55. Neef S, et al. CaMKII-dependent diastolic SR Ca2+ leak and elevated diastolic $\mathrm{Ca}^{2+}$ levels in right atrial myocardium of patients with atrial fibrillation. Circ Res. 2010;106(6):1134-1144.

56. Dikalov SI, et al. Nox2-induced production of mitochondrial superoxide in angiotensin II-mediated endothelial oxidative stress and hypertension. Antioxid Redox Signal. 2014;20(2):281-294.

57. Nazarewicz RR, Dikalova AE, Bikineyeva A, Dikalov SI. Nox2 as a potential target of mitochondrial superoxide and its role in endothelial oxidative stress. Am J Physiol Heart Circ Physiol. 2013;305(8):H1131-H1140.

58. Mustroph J, Neef S, Maier LS. CaMKII as a target for arrhythmia suppression. Pharmacol Ther. 2017;176:22-31

59. Mesubi OO, Anderson ME. Atrial remodelling in atrial fibrillation: CaMKII as a nodal proarrhythmic signal. Cardiovasc Res. 2016;109(4):542-557.

60. Tessier S, et al. Regulation of the transient outward $\mathrm{K}(+)$ current by $\mathrm{Ca}(2+) /$ calmodulin-dependent protein kinases II in human 
atrial myocytes. Circ Res. 1999;85(9):810-819.

61. Wagner S, et al. Ca/calmodulin kinase II differentially modulates potassium currents. Circ Arrhythm Electrophysiol. 2009;2(3):285-294.

62. Liang F, et al. Inhibitions of late INa and CaMKII act synergistically to prevent ATX-II-induced atrial fibrillation in isolated rat right atria. J Mol Cell Cardiol. 2016;94:122-130.

63. Fischer TH, et al. Late INa increases diastolic SR-Ca ${ }^{2+}-1$ eak in atrial myocardium by activating PKA and CaMKII. Cardiovasc Res. 2015;107(1):184-196.

64. Arora R, et al. Regional distribution of T-tubule density in left and right atria in dogs. Heart Rhythm. 2017;14(2):273-281.

65. Brandenburg S, et al. Axial tubule junctions control rapid calcium signaling in atria. J Clin Invest. 2016;126(10):3999-4015

66. Shah SJ, et al. Ultrastructural and cellular basis for the development of abnormal myocardial mechanics during the transition from hypertension to heart failure. Am J Physiol Heart Circ Physiol. 2014;306(1):H88-100.

67. Petitprez S, et al. SAP97 and dystrophin macromolecular complexes determine two pools of cardiac sodium channels Nav1.5 in cardiomyocytes. Circ Res. 2011;108(3):294-304.

68. Willis BC, Ponce-Balbuena D, Jalife J. Protein assemblies of sodium and inward rectifier potassium channels control cardiac excitability and arrhythmogenesis. Am J Physiol Heart Circ Physiol. 2015;308(12):H1463-H1473.

69. Saras J, Heldin CH. PDZ domains bind carboxy-terminal sequences of target proteins. Trends Biochem Sci. 1996;21(12):455-458

70. Anselmino M, Matta M, Castagno D, Giustetto C, Gaita F. Catheter ablation of atrial fibrillation in chronic heart failure: stateof-the-art and future perspectives. Europace. 2016;18(5):638-647.

71. Scott PA, Silberbauer J, Murgatroyd FD. The impact of adjunctive complex fractionated atrial electrogram ablation and linear lesions on outcomes in persistent atrial fibrillation: a meta-analysis. Europace. 2016;18(3):359-367.

72. Deshmukh A, et al. In-hospital complications associated with catheter ablation of atrial fibrillation in the United States between 2000 and 2010: analysis of 93801 procedures. Circulation. 2013;128(19):2104-2112.

73. Amit G, Kikuchi K, Greener ID, Yang L, Novack V, Donahue JK. Selective molecular potassium channel blockade prevents atrial fibrillation. Circulation. 2010;121(21):2263-2270.

74. Igarashi $\mathrm{T}$, et al. Connexin gene transfer preserves conduction velocity and prevents atrial fibrillation. Circulation. 2012;125(2):216-225.

75. Soucek R, et al. Genetic suppression of atrial fibrillation using a dominant-negative ether-a-go-go-related gene mutant. Heart Rhythm. 2012;9(2):265-272.

76. Aistrup G, et al. Non-viral gene-based inhibition of Gai/o-mediated vagal signaling in the posterior left atrium decreases vagal induced AF. Heart Rhythm. 2011;8(11):1722-1729.

77. Kunamalla A, et al. Constitutive expression of a dominant-negative TGF- $\beta$ Type II receptor in the posterior left atrium leads to beneficial remodeling of atrial fibrillation substrate. Circ Res. 2016;119(1):69-82.

78. Restrepo JG, Karma A. Spatiotemporal intracellular calcium dynamics during cardiac alternans. Chaos. 2009;19(3):037115.

79. Restrepo JG, Weiss JN, Karma A. Calsequestrin-mediated mechanism for cellular calcium transient alternans. Biophys J. 2008;95(8):3767-3789.

80. Grandi E, et al. Human atrial action potential and Ca2+ model: sinus rhythm and chronic atrial fibrillation. Circ Res 2011;109(9):1055-1066. 